\title{
MORE ON INTERVAL-VALUED INTUITIONISTIC FuZZY SOFT MULTI SETS
}

\author{
Anjan Mukherjee ${ }^{1}$, Ajoy Kanti Das ${ }^{2}$ and Abhijit Saha ${ }^{3}$ \\ ${ }^{1}$ Department of Mathematics,Tripura University, Agartala-799022,Tripura, INDIA, \\ ${ }^{2}$ Department of Mathematics, ICV-College, Belonia -799155, Tripura, INDIA, \\ ${ }^{3}$ Department of Mathematics, Techno India College, Agartala, Tripura, INDIA
}

\begin{abstract}
In 2013, Mukherje et al. developed the concept of interval-valued intuitionistic fuzzy soft multi set as a mathematical tool for making descriptions of the objective world more realistic, practical and accurate in some cases, making it very promising. In this paper we define some operations in interval-valued intuitionistic fuzzy soft multi set theory and show that the associative, distribution and De Morgan's type of results hold in interval-valued intuitionistic fuzzy soft multi set theory for the newly defined operations in our way. Also, we define the necessity and possibility operations on interval-valued intuitionistic fuzzy soft multi set theory and study their basic properties and some results.
\end{abstract}

\section{KEYWORDS}

Soft set, interval-valued intuitionistic fuzzy set, interval-valued intuitionistic fuzzy soft set, interval-valued intuitionistic fuzzy soft multi set.

\section{INTRODUCTION}

In recent years vague concepts have been used in different areas such as computer application, medical applications, information technology, pharmacology, economics and engineering since the classical mathematics methods are inadequate to solve many complex problems in these areas. In soft set theory there is no limited condition to the description of objects; so researchers can choose the form of parameters they need, which greatly simplifies the decision making process and make the process more efficient in the absence of partial information. Although many mathematical tools are available for modelling uncertainties such as probability theory, fuzzy set theory, rough set theory, interval valued mathematics etc, but there are inherent difficulties associated with each of these techniques. Moreover all these techniques lack in the parameterization of the tools and hence they could not be applied successfully in tackling problems especially in areas like economic, environmental and social problems domains. Soft set theory is standing in a unique way in the sense that it is free from the above difficulties.

In 1999, Molodstov [15] initiated the concept of soft set theory as a mathematical tool for dealing with uncertainties. Later on Maji et al.[14] presented some new definitions on soft sets such as subset, union, intersection and complements of soft sets and discussed in details the application of soft set in decision making problem. Based on the analysis of several operations on soft sets introduced in [15], Ali et al. [2] presented some new algebraic operations for soft sets and proved that certain De Morgan's law holds in soft set theory with respect to these new definitions. Combining soft sets [15] with fuzzy sets [23] and intuitionistic fuzzy sets [5], Maji et al. defined fuzzy soft sets [12] and intuitionistic fuzzy soft sets [13], which are rich potential for solving decision making problems. Basu et al. [9] defined some operation in fuzzy soft set and intuitionistic fuzzy soft set theory, such as extended intersection, restricted intersection, extended union and restricted union etc. and Bora et al. [10] presented "AND" and "OR" operators in intuitionistic fuzzy soft set theory and studied some results on intuitionistic fuzzy soft set theory.

DOI : $10.5121 /$ avc.2015.2201 
As a generalization of fuzzy set theory [23], intuitionistic fuzzy set theory [5] and interval-valued intuitionistic fuzzy set theory [6] makes descriptions of the objective world more realistic, practical and accurate in some cases, making it very promising. Jiang et al. [11] studied intervalvalued intuitionistic fuzzy soft sets and their properties.

Alkhazaleh and others [[1], [4], [7], [8], [22]] as a generalization of Molodtsov's soft set, presented the definition of a soft multi set and its basic operations such as complement, union, and intersection etc. In 2012, Alkhazaleh and other [3] introduced the concept of fuzzy soft multi set theory as a generalization of soft multi set theory and studied the application of fuzzy soft multi set based decision making problems and Mukherjee and Das [18] studied some results on fuzzy soft multiset theory. Recently, Mukherjee et al. [[19], [21]] constructed the fundamental theory on soft multi topological spaces and fuzzy soft multi topological spaces. Mukherjee and Das [[16], [17]] introduced the concepts of intuitionistic fuzzy soft multi sets and studied intuitionistic fuzzy soft multi topological spaces in details. Also they discussed the applications of intuitionistic fuzzy soft multi sets in information system. Mukherjee et al. [20] also introduced the concepts of interval valued intuitionistic fuzzy soft multi sets and studied their relation in details.

In fact all these concepts having a good application in other disciplines and real life problems are now catching momentum. But, it is seen that all these theories have their own difficulties. In this paper we have introduced some operations in interval-valued intuitionistic fuzzy soft multi set theory and show that the associative, distribution and De Morgan's type of results hold in interval-valued intuitionistic fuzzy soft multi set theory for the newly defined operations in our way. Also, we define the necessity and possibility operations on interval-valued intuitionistic fuzzy soft multi set theory and study their basic properties. Also some new results along with illustrating examples have been put forward in our work.

\section{PRELIMINARY NOTES}

In this section, we recall some basic notions in soft set theory, interval valued intuitionistic fuzzy set theory, interval valued intuitionistic fuzzy soft set theory and interval valued intuitionistic fuzzy soft multi set theory. Molodstov defined soft set in the following way. Let $U$ be an initial universe and $\mathrm{E}$ be a set of parameters. Let $\mathrm{P}(\mathrm{U})$ denotes the power set of $\mathrm{U}$ and $\mathrm{A} \subseteq \mathrm{E}$.

\section{Definition 2.1[15]}

A pair $(F, A)$ is called a soft set over $\mathrm{U}$, where $\mathrm{F}$ is a mapping given by $F: A \rightarrow P(U)$. In other words, soft set over $\mathrm{U}$ is a parameterized family of subsets of the universe $\mathrm{U}$.

\section{Definition 2.2[6]}

An interval valued intuitionistic fuzzy set $A$ over an universe set $U$ is defined as the object of the form $A=\left\{<x, \mu_{A}(x), \gamma_{A}(x)>: x \in U\right\}$, where $\mu_{A}: U \rightarrow$ Int $([0,1])$ and $\gamma_{\mathrm{A}}: U \rightarrow$ Int $([0,1])$ are functions such that the condition: $\forall \mathrm{x} \in \mathrm{U}, 0 \leq \sup \mu_{\mathrm{A}}(\mathrm{x})+\sup \gamma_{\mathrm{A}}(\mathrm{x}) \leq 1$ is satisfied, where Int $([0,1])$ denotes the set of all closed sub intervals of $[0,1]$.

The class of all interval valued intuitionistic fuzzy sets on $U$ is denoted by IVIFS(U).

\section{Definition 2.3 [11]}

A pair $(F, A)$ is called an interval valued intuitionistic fuzzy soft set over $U$, where $F$ is a mapping given by $\mathrm{F}: \mathrm{A} \rightarrow \operatorname{IVIFS}(\mathrm{U})$. For any parameter $\mathrm{a} \in \mathrm{A}, \mathrm{F}(\mathrm{a})$ can be written as

$$
F(a)=\left\{\frac{u}{\left(\left[\mu_{F(a)}^{L}(u), \mu_{F(a)}^{U}(u)\right],\left[v_{F(a)}^{L}(u), v_{F(a)}^{U}(u)\right]\right)}: u \in U\right\}
$$




\section{Definition 2.4[20]}

Let $\left\{U_{i}: i \in I\right\}$ be a collection of universes such that $\bigcap_{i \in I} U_{i}=\phi$ and let $\left\{E_{i}: i \in I\right\}$ be a collection of sets of parameters. Let $U=\prod_{i \in I} \operatorname{IVIFS}\left(U_{i}\right), E=\prod_{i \in I} E_{U_{i}}$ and $A \subseteq E$. A pair $(F, A)$ is called an interval valued intuitionistic fuzzy soft multi set over $\mathrm{U}$, where $\mathrm{F}$ is a mapping given by $F: A \rightarrow U$, such that $\forall a \in A$,

$$
F(a)=\left(\left\{\frac{u}{\left(\left[\mu_{F(a)}^{L}(u), \mu_{F(a)}^{U}(u)\right],\left[v_{F(a)}^{L}(u), v_{F(a)}^{U}(u)\right]\right)}: u \in U_{i}\right\}: i \in I\right) .
$$

\section{Definition 2.5[20]}

Let $(F, A)$ and $(G, B)$ be two interval valued intuitionistic fuzzy soft multisets over $U$. Then $(F$, A) is called an interval valued intuitionistic fuzzy soft multi subset of $(\mathrm{G}, \mathrm{B})$ if

(a) $A \subseteq B$ and

(b) $\forall e_{U_{i}, j} \in a_{k},\left(e_{U_{i}, j}, F_{e_{U_{i}, j}}\right)$ is an interval valued intuitionistic fuzzy subset of $\left(e_{U_{i}, j}, G_{e_{U_{i}, j}}\right)$, where $a_{k} \in A, \mathrm{k} \in\{1,2,3, . ., \mathrm{n}\}, i \in\{1,2,3, . ., \mathrm{m}\}$ and $j \in\{1,2,3, . ., \mathrm{r}\}$. This relationship is denoted by $(\mathrm{F}, \mathrm{A}) \tilde{\subseteq}(\mathrm{G}, \mathrm{B})$

\section{Definition 2.6[20]}

The complement of an interval valued intuitionistic fuzzy soft multi set $(F, A)$ over $\mathrm{U}$ is denoted by $(F, A)^{c}$ and is defined by $(F, A)^{c}=\left(F^{c}, A\right)$, where $\forall a \in A$,

$$
F^{c}(a)=\left(\left\{\frac{u}{\left(\left[v_{F(a)}^{L}(u), v_{F(a)}^{U}(u)\right],\left[\mu_{F(a)}^{L}(u), \mu_{F(a)}^{U}(u)\right]\right)}: u \in U_{i}\right\}: i \in I\right)
$$

\section{Definition 2.7[20]}

An interval valued intuitionistic fuzzy soft multiset $(\mathrm{F}, \mathrm{A})$ over $\mathrm{U}$ is called a null interval valued intuitionistic fuzzy soft multiset, denoted by $(\mathrm{F}, \mathrm{A})_{\Phi}$, if all the interval valued intuitionistic fuzzy soft multiset parts of (F, A) equals $\phi$.

\section{Definition 2.8[20]}

An interval valued intuitionistic fuzzy soft multiset $(F, A)$ over $U$ is called an absolute interval valued intuitionistic fuzzy soft multiset, denoted by $(\mathrm{F}, \mathrm{A})_{U}$, if $\left(e_{U_{i}, j}, F_{e_{U_{i}, j}}\right)=U_{i}, \forall i$.

\section{Definition 2.9[20]}

The union of two interval valued intuitionistic fuzzy soft multisets $(\mathrm{F}, A)$ and $(\mathrm{G}, B)$ over $U$ is an interval valued intuitionistic fuzzy soft multiset $(\mathrm{H}, \mathrm{D})$ where $D=A \cup B$ and $\forall e \in D$,

$$
H(e)= \begin{cases}F(e), & \text { if } e \in A-B \\ G(e), & \text { if } e \in B-A \\ \cup(F(e), G(e)), & \text { if } e \in A \cap B\end{cases}
$$


Where $U(F(e), G(e))=F_{e_{U_{i}, j}} \cup G_{e_{U_{i}, j}} \forall i \in\{1,2,3, . ., \mathrm{m}\} \quad$ with $\quad \cup \quad$ as an interval valued intuitionistic fuzzy union and is written as $(F, A) \tilde{\cup}(G, B)=(H, D)$

\section{Definition 2.10[20]}

The intersection of two interval valued intuitionistic fuzzy soft multisets $(\mathrm{F}, A)$ and $(\mathrm{G}, B)$ over $U$ is an interval valued intuitionistic fuzzy soft multiset $(\mathrm{H}, \mathrm{D})$ where

$D=A \cap B$ and $\forall e \in D$,

$H(e)= \begin{cases}F(e), & \text { if } e \in A-B \\ G(e), & \text { if } e \in B-A \\ \cap(F(e), G(e)), & \text { if } e \in A \cap B\end{cases}$

where $\bigcap(F(e), G(e))=F_{e_{U_{i}, j}} \cap G_{e_{U_{i}, j}} \forall i \in\{1,2,3, \ldots, \mathrm{n}\} \quad$ with $\cap \quad$ as an interval valued intuitionistic fuzzy intersection and is written as $(F, A) \tilde{\cap}(G, B)=(H, C)$

\section{SOME RESULTS ON INTERVAL-VALUED INTUITIONISTIC FUZZY SOFT MULTI SETS}

\section{Definition 3.1}

An interval-valued intuitionistic fuzzy soft multi set $(F, A)$ over $\mathrm{U}$ is called an interval valued intuitionistic fuzzy soft multi subset of an interval valued intuitionistic fuzzy soft multi set $(G, B)$ if

(a) $A \subseteq B$ and

(b) $\forall e \in A, F(e) \subseteq G(e) \Leftrightarrow \mu_{F(e)}^{L}(u) \leq \mu_{G(e)}^{L}(u), \mu_{F(e)}^{U}(u) \leq \mu_{G(e)}^{U}(u)$ and $v_{F(e)}^{L}(u) \geq v_{G(e)}^{L}(u), v_{F(e)}^{U}(u) \geq v_{G(e)}^{U}(u)$ $\forall u \in U_{i}, i \in I$ and this relationship is denoted by $(F, A) \tilde{\subseteq}(G, B)$.

\section{Definition 3.2}

The restricted union of two interval-valued intuitionistic fuzzy soft multi sets $(F, A)$ and $(G, B)$ over $\mathrm{U}$ is an interval-valued intuitionistic fuzzy soft multi set $(H, C)$, where $C=A \cap B$ and $\forall e \in C, H(e)=\cup(F(e), G(e))$

$=\left(\left\{\frac{u}{\left(\left[\max \left\{\mu_{F(e)}^{L}(u), \mu_{G(e)}^{L}(u)\right\}, \max \left\{\mu_{F(e)}^{U}(u), \mu_{G(e)}^{U}(u)\right\}\right],\left[\min \left\{v_{F(e)}^{L}(u), v_{G(e)}^{L}(u)\right\}, \min \left\{v_{F(e)}^{U}(u), v_{G(e)}^{U}(u)\right\}\right]\right)}: u \in U_{i}\right\}: i \in I\right) \mathrm{a}$ nd is written as $(F, A) \tilde{\cup}_{R}(G, B)=(H, C)$.

\section{Definition 3.3}

The restricted intersection of two interval-valued intuitionistic fuzzy soft multi sets $(F, A)$ and $(G$, $B$ ) over $\mathrm{U}$ is an interval-valued intuitionistic fuzzy soft multi set $(H, D)$ where $D=A \cap B$ and $\forall e \in D, H(e)=\bigcap(F(e), G(e))$

$=\left(\left\{\frac{u}{\left(\left[\min \left\{\mu_{F(e)}^{L}(u), \mu_{G(e)}^{L}(u)\right\}, \min \left\{\mu_{F(e)}^{U}(u), \mu_{G(e)}^{U}(u)\right\}\right],\left[\max \left\{v_{F(e)}^{L}(u), v_{G(e)}^{L}(u)\right\}, \max \left\{v_{F(e)}^{U}(u), \nu_{G(e)}^{U}(u)\right\}\right]\right)}: u \in U_{i}\right\}: i \in I\right)$

and is written as $(F, A) \tilde{\cap}_{R}(G, B)=(H, D)$. 


\section{Example 3.4}

Let us consider there are two universes $U_{1}=\left\{h_{1}, h_{2}, h_{3}\right\}, U_{2}=\left\{c_{1}, c_{2}\right\}$ and let $\left\{E_{U_{1}}, E_{U_{2}}\right\}$ be a collection of sets of decision parameters related to the above universes, where

$E_{U_{1}}=\left\{e_{U_{1}, 1}=\right.$ expensive, $e_{U_{1}, 2}=$ cheap, $e_{U_{1}, 3}=$ wooden $\}$

$E_{U_{1}}=\left\{e_{U_{2}, 1}=\right.$ beautiful, $e_{U_{2}, 2}=$ model, $e_{U_{2}, 3}=$ sporty $\}$,

Let $U=\prod_{i=1}^{2} F S\left(U_{i}\right), E=\prod_{i=1}^{2} E_{U_{i}}$ and

$A=\left\{e_{1}=\left(e_{U_{1}, 1}, e_{U_{2}, 1}\right), e_{2}=\left(e_{U_{1}, 1}, e_{U_{2}, 2}\right), e_{3}=\left(e_{U_{1}, 2}, e_{U_{2}, 1}\right)\right\}$,

$B=\left\{e_{1}=\left(e_{U_{1}, 1}, e_{U_{2}, 1}\right), e_{2}=\left(e_{U_{1}, 1}, e_{U_{2}, 2}\right), e_{4}=\left(e_{U_{1}, 3}, e_{U_{2}, 2}\right)\right\}$.

Suppose that

$$
\begin{aligned}
&(F, A)=\{\left(e_{1},\left(\left\{\frac{h_{1}}{([0.2,0.3],[0.3,0.4])}, \frac{h_{2}}{([0.4,0.5][0.4,0.5])}, \frac{h_{3}}{([0.8,0.9],[0,0.1])}\right\},\left\{\frac{c_{1}}{([0.7,0.8],[0.1,0.2])}, \frac{c_{2}}{([0.4,0.5],[0.3,0.4])}\right\}\right)\right), \\
&\left(e_{2},\left(\left\{\frac{h_{1}}{([0.8,0.9],[0,0.1])}, \frac{h_{2}}{([0,7,0.8],[0.1,0.2])}, \frac{h_{3}}{([1,1],[0,0])}\right\},\left\{\frac{c_{1}}{([0.8,0.8],[0.1,0.1])}, \frac{c_{2}}{([0.4,0.5][0.3,0.5])}\right\}\right)\right), \\
&\left(e_{3},\left(\left\{\frac{h_{1}}{([0.7,0.8],[0.1,0.2])}, \frac{h_{2}}{([0.6,0.7],[0.1,0.3])}, \frac{h_{3}}{([1,1],[0,0])}\right\},\left\{\frac{c_{1}}{([0.2,0.3],[0.3,0.4])}, \frac{c_{2}}{([0.4,0.5][0.2,0.3])}\right\}\right)\right\}, \\
&(G, B)=\left\{\left(e_{1},\left(\left\{\frac{h_{1}}{([0.4,0.5][0.3,0.4])}, \frac{h_{2}}{([0,7,0.8],[0.1,0.2])}, \frac{h_{3}}{([0.8,0.8],[0.1,0.1])}\right\},\left\{\frac{c_{1}}{([0,7,0.8],[0.1,0.2])}, \frac{c_{2}}{([0.6,0.7],[0.1,0.3])}\right\}\right)\right),\right. \\
&\left(e_{2},\left\{\left\{\frac{h_{1}}{([0.4,0.5][0.2,0.5])}, \frac{h_{2}}{([0.8,0.9],[0,0.1])}, \frac{h_{3}}{([1,1],[0,0])}\right\},\left\{\frac{c_{1}}{([0.4,0.5][0.2,0.3])}, \frac{1}{([0.6,0.7],[0.1,0.3])}\right\}\right)\right), \\
&\left.\left(e_{4},\left(\left\{\frac{h_{1}}{([0.8,0.8],[0.1,0.1])}, \frac{h_{2}}{([0.2,0.3],[0.3,0.4])}, \frac{h_{3}}{([1,1],[0,0])}\right\},\left\{\frac{c_{1}}{([0.8,0.8],[0.1,0.1])}, \frac{c_{2}}{([0,7,0.8],[0.1,0.2])}\right\}\right)\right)\right\}
\end{aligned}
$$

be two interval-valued intuitionistic fuzzy soft multi sets over $\mathrm{U}$, then we have,

(i). $(F, A) \tilde{\cup}_{R}(G, B)$

$$
\begin{aligned}
=\left\{\left(e_{1},\left(\left\{\frac{h_{1}}{([0.4,0.5],[0.3,0.4])}, \frac{h_{2}}{([0.7,0.8][0.1,0.2])}, \frac{h_{3}}{([0.8,0.9],[0,0.1])}\right\},\left\{\frac{c_{1}}{([0.7,0.8],[0.1,0.2])}, \frac{c_{2}}{([0.6,0.7],[0.1,0.3])}\right\}\right),\right.\right. \\
\left.\left(e_{2},\left(\left\{\frac{h_{1}}{([0.8,0.9],[0,0.1])}, \frac{h_{2}}{([0.8,0.9],[0,0.1])}, \frac{h_{3}}{([1,1],[0,0])}\right\},\left\{\frac{c_{1}}{([0.8,0.8],[0.1,0.1])}, \frac{c_{2}}{([0.6,0.7],[0.1,0.3])}\right\}\right)\right)\right\}
\end{aligned}
$$

(ii). $(F, A) \tilde{\cap}_{R}(G, B)$

$$
\begin{gathered}
=\left\{\left(e_{1},\left(\left\{\frac{h_{1}}{([0.2,0.3],[0.3,0.4])}, \frac{h_{2}}{([0.4,0.5][0.4,0.5])}, \frac{h_{3}}{([0.8,0.8],[0.1,0.1])}\right\},\left\{\frac{c_{1}}{([0.7,0.8],[0.1,0.2])}, \frac{c_{2}}{([0.4,0.5],[0.3,0.4])}\right\}\right),\right.\right. \\
\left.\left(e_{2},\left(\left\{\frac{h_{1}}{([0.4,0.5][0.2,0.5])}, \frac{h_{2}}{([0,7,0.8],[0.1,0.2])}, \frac{h_{3}}{([1,1],[0,0])}\right\},\left\{\frac{c_{1}}{([0.4,0.5][0.2,0.3])}, \frac{c_{2}}{([0.4,0.5][0.3,0.5])}\right\}\right)\right)\right\}
\end{gathered}
$$

\section{Proposition 3.5 (Associative Laws)}

Let $(\mathrm{F}, \mathrm{A}),(\mathrm{G}, \mathrm{B})$ and $(\mathrm{H}, \mathrm{C})$ are three interval valued intuitionistic fuzzy soft multi sets over $\mathrm{U}$, then we have the following properties:
1. $(F, A) \tilde{\cap}_{R}\left((G, B) \tilde{\cap}_{R}(H, C)\right)=\left((F, A) \tilde{\cap}_{R}(G, B)\right) \tilde{\cap}_{R}(H, C)$
2. $(F, A) \tilde{\cup}_{R}\left((G, B) \tilde{\cup}_{R}(H, C)\right)=\left((F, A) \tilde{\cup}_{R}(G, B)\right) \tilde{\cup}_{R}(H, C)$ 
Proof. 1. Assume that $(G, B) \tilde{\cap}_{R}(H, C)=(I, D)$, where $D=B \cap C$ and $\forall e \in D, I(e)=G(e) \cap H(e)$

$$
\begin{aligned}
= & \left(\left\{\frac{u}{\left(\left[\min \left\{\mu_{G(e)}^{L}(u), \mu_{H(e)}^{L}(u)\right\}, \min \left\{\mu_{G(e)}^{U}(u), \mu_{H(e)}^{U}(u)\right\}\right],\left[\max \left\{v_{G(e)}^{L}(u), v_{H(e)}^{L}(u)\right\}, \max \left\{v_{G(e)}^{U}(u), v_{H(e)}^{U}(u)\right\}\right]\right)}:\right.\right. \\
& \left.\left.u \in U_{i}\right\}: i \in I\right)
\end{aligned}
$$

Since $(F, A) \tilde{\cap}_{R}\left((G, B) \tilde{\cap}_{R}(H, C)\right)=(F, A) \tilde{\cap}_{R}(I, D)$, we suppose that $(F, A) \tilde{\cap}_{R}(I, D)=(K, M)$ where $M=A \cap D=A \cap B \cap C$ and $\forall e \in M$,

$$
\begin{aligned}
& \qquad K(e)=F(e) \cap I(e)=\left(\left\{\frac{u}{\left(\mu_{K(e)}(u), v_{K(e)}(u)\right)}: u \in U_{i}\right\}: i \in I\right) \\
& \text { where } \mu_{K(e)}(u)=\left[\min \left\{\mu_{F(e)}^{L}(u), \mu_{I(e)}^{L}(u)\right\}, \min \left\{\mu_{F(e)}^{U}(u), \mu_{I(e)}^{U}(u)\right\}\right] \\
& =\left[\min \left\{\mu_{F(e)}^{L}(u), \min \left\{\mu_{G(e)}^{L}(u), \mu_{H(e)}^{L}(u)\right\}\right\}, \min \left\{\mu_{F(e)}^{U}(u), \min \left\{\mu_{G(e)}^{U}(u), \mu_{H(e)}^{U}(u)\right\}\right\}\right] \\
& =\left[\min \left\{\mu_{F(e)}^{L}(u), \mu_{G(e)}^{L}(u), \mu_{H(e)}^{L}(u)\right\}, \min \left\{\mu_{F(e)}^{U}(u), \mu_{G(e)}^{U}(u), \mu_{H(e)}^{U}(u)\right\}\right] \\
& \text { and } v_{K(e)}(u)=\left[\max \left\{v_{F(e)}^{L}(u), v_{I(e)}^{L}(u)\right\}, \max \left\{v_{F(e)}^{U}(u), v_{I(e)}^{U}(u)\right\}\right] \\
& =\left[\max \left\{v_{F(e)}^{L}(u), \max \left\{v_{G(e)}^{L}(u), v_{H(e)}^{L}(u)\right\}\right\}, \max \left\{v_{F(e)}^{U}(u), \max \left\{v_{G(e)}^{U}(u), v_{H(e)}^{U}(u)\right\}\right\}\right] \\
& =\left[\max \left\{v_{F(e)}^{L}(u), v_{G(e)}^{L}(u), v_{H(e)}^{L}(u)\right\}, \max \left\{v_{F(e)}^{U}(u), v_{G(e)}^{U}(u), v_{H(e)}^{U}(u)\right\}\right] .
\end{aligned}
$$

Suppose that $(F, A) \tilde{\cap}_{R}(G, B)=(J, N)$, where $N=A \cap B$ and $\forall e \in N$,

$$
\begin{aligned}
J(e) & =F(e) \cap G(e)=\left(\left\{\frac{u}{\left(\mu_{J(e)}(u), v_{J(e)}(u)\right)}: u \in U_{i}\right\}: i \in I\right) \\
\mu_{J(e)}(u) & =\left[\min \left\{\mu_{F(e)}^{L}(u), \mu_{G(e)}^{L}(u)\right\}, \min \left\{\mu_{F(e)}^{U}(u), \mu_{G(e)}^{U}(u)\right\}\right], \\
v_{J(e)}(u) & =\left[\max \left\{v_{F(e)}^{L}(u), v_{G(e)}^{L}(u)\right\}, \max \left\{v_{F(e)}^{U}(u), v_{G(e)}^{U}(u)\right\}\right]
\end{aligned}
$$

Since $\left((F, A) \tilde{\cap}_{R}(G, B)\right) \tilde{\cap}_{R}(H, C)=(J, N) \tilde{\cap}_{R}(H, C)$, we suppose that $(J, N) \tilde{\cap}_{R}(H, C)=(O, N \cap C)$ where

$$
\begin{aligned}
\forall e \in N \cap C= & A \cap B \cap C, O(e)=J(e) \cap H(e)=\left(\left\{\frac{u}{\left(\mu_{O(e)}(u), v_{O(e)}(u)\right)}: u \in U_{i}\right\}: i \in I\right) \\
& \mu_{O(e)}(u)=\left[\min \left\{\mu_{J(e)}^{L}(u), \mu_{H(e)}^{L}(u)\right\}, \min \left\{\mu_{J(e)}^{U}(u), \mu_{H(e)}^{U}(u)\right\}\right] \\
& =\left[\min \left\{\min \left\{\mu_{F(e)}^{L}(u), \mu_{G(e)}^{L}(u)\right\}, \mu_{H(e)}^{L}(u)\right\}, \min \left\{\min \left\{\mu_{F(e)}^{U}(u), \mu_{G(e)}^{U}(u)\right\}, \mu_{H(e)}^{U}(u)\right\}\right] \\
& =\left[\min \left\{\mu_{F(e)}^{L}(u), \mu_{G(e)}^{L}(u), \mu_{H(e)}^{L}(u)\right\}, \min \left\{\mu_{F(e)}^{U}(u), \mu_{G(e)}^{U}(u), \mu_{H(e)}^{U}(u)\right\}\right]=\mu_{K(e)}(u)
\end{aligned}
$$

and

$$
\begin{aligned}
& v_{O(e)}(u)=\left[\max \left\{v_{J(e)}^{L}(u), v_{H(e)}^{L}(u)\right\}, \max \left\{v_{J(e)}^{U}(u), v_{H(e)}^{U}(u)\right\}\right] \\
& =\left[\max \left\{\max \left\{v_{F(e)}^{L}(u), v_{G(e)}^{L}(u)\right\}, v_{H(e)}^{L}(u)\right\}, \max \left\{\max \left\{v_{F(e)}^{U}(u), v_{G(e)}^{U}(u)\right\}, v_{H(e)}^{U}(u)\right\}\right] \\
& =\left[\max \left\{v_{F(e)}^{L}(u), v_{G(e)}^{L}(u), v_{H(e)}^{L}(u)\right\}, \max \left\{v_{F(e)}^{U}(u), v_{G(e)}^{U}(u), v_{H(e)}^{U}(u)\right\}\right]=v_{K(e)}(u)
\end{aligned}
$$

Consequently, $\mathrm{K}$ and $\mathrm{O}$ are the same operators. Thus

$$
(F, A) \tilde{\cap}_{R}\left((G, B) \tilde{\cap}_{R}(H, C)\right)=\left((F, A) \tilde{\cap}_{R}(G, B)\right) \tilde{\cap}_{R}(H, C) .
$$

The proof of (2) is similar to that of (1).

\section{Proposition 3.5 (Distributive Laws)}

Let $(\mathrm{F}, \mathrm{A}),(\mathrm{G}, \mathrm{B})$ and $(\mathrm{H}, \mathrm{C})$ are three interval valued intuitionistic fuzzy soft multi sets over $\mathrm{U}$, then we have the following properties: 
(1). $(F, A) \tilde{\cup}_{R}\left((G, B) \tilde{\cap}_{R}(H, C)\right)=\left((F, A) \tilde{\cup}_{R}(G, B)\right) \tilde{\cap}_{R}\left((F, A) \tilde{\cup}_{R}(H, C)\right)$

(2). $(F, A) \tilde{\cap}_{R}\left((G, B) \tilde{\cup}_{R}(H, C)\right)=\left((F, A) \tilde{\cap}_{R}(G, B)\right) \tilde{\cup}_{R}\left((F, A) \tilde{\cap}_{R}(H, C)\right)$

Proof. (1). Assume that $(G, B) \tilde{\cap}_{R}(H, C)=(I, D)$, where $D=B \cap C$ and $\forall e \in D$,

$I(e)=G(e) \cap H(e)$

$$
\begin{aligned}
= & \left(\left\{\frac{u}{\left(\left[\min \left\{\mu_{G(e)}^{L}(u), \mu_{H(e)}^{L}(u)\right\}, \min \left\{\mu_{G(e)}^{U}(u), \mu_{H(e)}^{U}(u)\right\}\right],\left[\max \left\{v_{G(e)}^{L}(u), \nu_{H(e)}^{L}(u)\right\}, \max \left\{v_{G(e)}^{U}(u), v_{H(e)}^{U}(u)\right\}\right]\right)}:\right.\right. \\
& \left.\left.u \in U_{i}\right\}: i \in I\right)
\end{aligned}
$$

Since $(F, A) \tilde{\cup}_{R}\left((G, B) \tilde{\cap}_{R}(H, C)\right)=(F, A) \tilde{\cup}_{R}(I, D)$, we suppose that $(F, A) \tilde{\cup}_{R}(I, D)=(K, M)$ where $M=A \cap D=A \cap B \cap C$ and $\forall e \in M$,

$K(e)=F(e) \cup I(e)=\left(\left\{\frac{u}{\left(\mu_{K(e)}(u), v_{K(e)}(u)\right)}: u \in U_{i}\right\}: i \in I\right)$

where $\mu_{K(e)}(u)=\left[\max \left\{\mu_{F(e)}^{L}(u), \mu_{I(e)}^{L}(u)\right\}, \max \left\{\mu_{F(e)}^{U}(u), \mu_{I(e)}^{U}(u)\right\}\right]$

$=\left[\max \left\{\mu_{F(e)}^{L}(u), \min \left\{\mu_{G(e)}^{L}(u), \mu_{H(e)}^{L}(u)\right\}\right\}, \max \left\{\mu_{F(e)}^{U}(u), \min \left\{\mu_{G(e)}^{U}(u), \mu_{H(e)}^{U}(u)\right\}\right\}\right]$

$=\left[\min \left\{\max \left\{\mu_{F(e)}^{L}(u), \mu_{G(e)}^{L}(u)\right\}, \max \left\{\mu_{F(e)}^{L}(u), \mu_{H(e)}^{L}(u)\right\}\right\}\right.$,

$\left.\min \left\{\max \left\{\mu_{F(e)}^{U}(u), \mu_{G(e)}^{U}(u)\right\}, \max \left\{\mu_{F(e)}^{U}(u), \mu_{H(e)}^{U}(u)\right\}\right\}\right]$

and $v_{K(e)}(u)=\left[\min \left\{v_{F(e)}^{L}(u), v_{I(e)}^{L}(u)\right\}, \min \left\{v_{F(e)}^{U}(u), v_{I(e)}^{U}(u)\right\}\right]$

$=\left[\min \left\{v_{F(e)}^{L}(u), \max \left\{v_{G(e)}^{L}(u), v_{H(e)}^{L}(u)\right\}\right\}, \min \left\{v_{F(e)}^{U}(u), \max \left\{v_{G(e)}^{U}(u), v_{H(e)}^{U}(u)\right\}\right\}\right]$

$=\left[\max \left\{\min \left\{v_{F(e)}^{L}(u), v_{G(e)}^{L}(u)\right\}, \min \left\{v_{F(e)}^{L}(u), v_{H(e)}^{L}(u)\right\}\right\}\right.$,

$\left.\max \left\{\min \left\{v_{F(e)}^{U}(u), v_{G(e)}^{U}(u)\right\}, \min \left\{v_{F(e)}^{U}(u), v_{H(e)}^{U}(u)\right\}\right\}\right]$.

Suppose that $(F, A) \tilde{\cup}_{R}(G, B)=(J, N)$, where $N=A \cap B$ and $\forall e \in N$,

$J(e)=F(e) \cup G(e)=\left(\left\{\frac{u}{\left(\mu_{J(e)}(u), v_{J(e)}(u)\right)}: u \in U_{i}\right\}: i \in I\right)$

$\mu_{J(e)}(u)=\left[\max \left\{\mu_{F(e)}^{L}(u), \mu_{G(e)}^{L}(u)\right\}, \max \left\{\mu_{F(e)}^{U}(u), \mu_{G(e)}^{U}(u)\right\}\right]$,

$v_{J(e)}(u)=\left[\min \left\{v_{F(e)}^{L}(u), v_{G(e)}^{L}(u)\right\}, \min \left\{v_{F(e)}^{U}(u), v_{G(e)}^{U}(u)\right\}\right]$

Again, let $(F, A) \tilde{\cup}_{R}(H, C)=(S, T)$, where $T=A \cap C$ and $\forall e \in T$,

$S(e)=F(e) \cup H(e)=\left(\left\{\frac{u}{\left(\mu_{S(e)}(u), v_{S(e)}(u)\right)}: u \in U_{i}\right\}: i \in I\right)$

$\mu_{S(e)}(u)=\left[\max \left\{\mu_{F(e)}^{L}(u), \mu_{H(e)}^{L}(u)\right\}, \max \left\{\mu_{F(e)}^{U}(u), \mu_{H(e)}^{U}(u)\right\}\right]$,

$v_{S(e)}(u)=\left[\min \left\{v_{F(e)}^{L}(u), v_{H(e)}^{L}(u)\right\}, \min \left\{v_{F(e)}^{U}(u), v_{H(e)}^{U}(u)\right\}\right]$

Since $\left((F, A) \tilde{\cup}_{R}(G, B)\right) \tilde{\cap}_{R}\left((F, A) \tilde{\cup}_{R}(H, C)\right)=(J, N) \tilde{\cap}_{R}(S, T)$, we suppose that

$(J, N) \tilde{\cap}_{R}(S, T)=(O, N \cap T)$ where $\forall e \in N \cap T=A \cap B \cap C$,

$O(e)=J(e) \cap S(e)=\left(\left\{\frac{u}{\left(\mu_{O(e)}(u), v_{O(e)}(u)\right)}: u \in U_{i}\right\}: i \in I\right)$ 


$$
\begin{aligned}
& \mu_{O(e)}(u)=\left[\min \left\{\mu_{J(e)}^{L}(u), \mu_{S(e)}^{L}(u)\right\}, \min \left\{\mu_{J(e)}^{U}(u), \mu_{S(e)}^{U}(u)\right\}\right] \\
& =\left[\min \left\{\max \left\{\mu_{F(e)}^{L}(u), \mu_{G(e)}^{L}(u)\right\}, \max \left\{\mu_{F(e)}^{L}(u), \mu_{H(e)}^{L}(u)\right\}\right\},\right. \\
& \left.\quad \min \left\{\max \left\{\mu_{F(e)}^{U}(u), \mu_{G(e)}^{U}(u)\right\}, \max \left\{\mu_{F(e)}^{U}(u), \mu_{H(e)}^{U}(u)\right\}\right\}\right]=\mu_{K(e)}(u) \\
& \text { and } \\
& v_{O(e)}(u)=\left[\max \left\{v_{J(a, b)}^{L}(u), v_{H(c)}^{L}(u)\right\}, \max \left\{v_{J(a, b)}^{U}(u), v_{H(c)}^{U}(u)\right\}\right] \\
& =\left[\max \left\{\min \left\{v_{F(e)}^{L}(u), v_{G(e)}^{L}(u)\right\}, \min \left\{v_{F(e)}^{U}(u), v_{G(e)}^{U}(u)\right\}\right\},\right. \\
& \left.\quad \max \left\{\min \left\{v_{F(e)}^{L}(u), v_{H(e)}^{L}(u)\right\}, \min \left\{v_{F(e)}^{U}(u), v_{H(e)}^{U}(u)\right\}\right\}\right]=v_{K(e)}(u)
\end{aligned}
$$

Consequently, $\mathrm{K}$ and $\mathrm{O}$ are the same operators. Thus

$(F, A) \tilde{\cap}_{R}\left((G, B) \tilde{\cap}_{R}(H, C)\right)=\left((F, A) \tilde{\cap}_{R}(G, B)\right) \tilde{\cap}_{R}(H, C)$.

The proof of (2) is similar to that of (1).

\section{Proposition 3.6 (De Morgan Laws)}

For two interval-valued intuitionistic fuzzy soft multi sets $(\mathrm{F}, \mathrm{A})$ and $(\mathrm{G}, \mathrm{B})$ over $\mathrm{U}$, then we have

$$
\begin{aligned}
& \text { [1]. }\left((F, A) \tilde{\cup}_{R}(G, B)\right)^{c}=(F, A)^{c} \tilde{\cap}_{R}(G, B)^{c} \\
& \text { [2]. }\left((F, A) \tilde{\cap}_{R}(G, B)\right)^{c}=(F, A)^{c} \tilde{\cup}_{R}(G, B)^{c}
\end{aligned}
$$

Proof. [1]. Let $(F, A) \tilde{\cup}_{R}(G, B)=(H, C)$, where $C=A \cap B$ and $\forall e \in C$,

$H(e)=\bigcup(F(e), G(e))=$

$\int\left\{\frac{u}{\left(\left[\max \left\{\mu_{F(a)}^{L}(u), \mu_{G(a)}^{L}(u)\right\}, \max \left\{\mu_{F(a)}^{U}(u), \mu_{G(a)}^{U}(u)\right\}\right],\left[\min \left\{v_{F(a)}^{L}(u), v_{G(a)}^{L}(u)\right\}, \min \left\{v_{F(a)}^{U}(u), v_{G(a)}^{U}(u)\right\}\right]\right)}:\right.$

$\left.\left.u \in U_{i}\right\}: i \in I\right)$. Thus $\left((F, A) \tilde{\cup}_{R}(G, B)\right)^{c}=(H, C)^{c}=\left(H^{c}, C\right)$, where $C=A \cap B$ and $\forall e \in C$,

$H^{c}(e)=$

$\int\left\{\frac{u}{\left(\left[\min \left\{v_{F(a)}^{L}(u), v_{G(a)}^{L}(u)\right\}, \min \left\{v_{F(a)}^{U}(u), v_{G(a)}^{U}(u)\right\}\right],\left[\max \left\{\mu_{F(a)}^{L}(u), \mu_{G(a)}^{L}(u)\right\}, \max \left\{\mu_{F(a)}^{U}(u), \mu_{G(a)}^{U}(u)\right\}\right]\right)}:\right.$

$\left.\left.u \in U_{i}\right\}: i \in I\right)$. Again, $(F, A)^{c} \tilde{\cap}_{R}(G, B)^{c}=(K, D)$, where $D=A \cap B$ and $\forall e \in D$,

$K(e)=\bigcap\left(F^{c}(e), G^{c}(e)\right)=$

$\int\left\{\frac{u}{\left(\left[\min \left\{v_{F(a)}^{L}(u), v_{G(a)}^{L}(u)\right\}, \min \left\{v_{F(a)}^{U}(u), v_{G(a)}^{U}(u)\right\}\right],\left[\max \left\{\mu_{F(a)}^{L}(u), \mu_{G(a)}^{L}(u)\right\}, \max \left\{\mu_{F(a)}^{U}(u), \mu_{G(a)}^{U}(u)\right\}\right]\right)}:\right.$

$\left.\left.u \in U_{i}\right\}: i \in I\right)$.

e see that $\mathrm{C}=\mathrm{D}$ and $\forall e \in C, H^{c}(e)=K(e)$. Hence the result.

The other can be proved similarly.

\section{Definition 3.7}

The extended union of two interval-valued intuitionistic fuzzy soft multi sets (F, A) and (G, B) over $\mathrm{U}$ is an intuitionistic fuzzy soft multi set $(\mathrm{H}, \mathrm{D})$, where $D=A \cup B$ and $\forall e \in D$,

$$
\begin{gathered}
H(e)= \begin{cases}F(e), & \text { if } e \in A-B \\
G(e), & \text { if } e \in B-A \\
\cup(F(e), G(e)), & \text { if } e \in A \cap B,\end{cases} \\
\text { where } U(F(e), G(e))=\left(\left\{\frac{u}{\left(\mu_{H(e)}(u), v_{H(e)}(u)\right)}: u \in U_{i}\right\}: i \in I\right)
\end{gathered}
$$


$\mu_{H(e)}(u)=\left[\max \left\{\mu_{F(a)}^{L}(u), \mu_{G(a)}^{L}(u)\right\}, \max \left\{\mu_{F(a)}^{U}(u), \mu_{G(a)}^{U}(u)\right\}\right]$

and $v_{H(e)}(u)=\left[\min \left\{v_{F(a)}^{L}(u), v_{G(a)}^{L}(u)\right\}, \min \left\{v_{F(a)}^{U}(u), v_{G(a)}^{U}(u)\right\}\right]$

and is written as $(F, A) \tilde{\cup}_{E}(G, B)=(H, D)$.

\section{Definition 3.8}

The extended intersection of two interval-valued intuitionistic fuzzy soft multi sets $(F, A)$ and $(G$, $B$ ) over $\mathrm{U}$ is an intuitionistic fuzzy soft multi set $(H, D)$, where $D=A \cup B$ and $\forall e \in D$,

$$
H(e)= \begin{cases}F(e), & \text { if } e \in A-B \\ G(e), & \text { if } e \in B-A \\ \cap(F(e), G(e)), & \text { if } e \in A \cap B\end{cases}
$$

where $\bigcap(F(e), G(e))=\left(\left\{\frac{u}{\left(\mu_{H(e)}(u), v_{H(e)}(u)\right)}: u \in U_{i}\right\}: i \in I\right)$

$\mu_{H(e)}(u)=\left[\max \left\{\mu_{F(a)}^{L}(u), \mu_{G(a)}^{L}(u)\right\}, \max \left\{\mu_{F(a)}^{U}(u), \mu_{G(a)}^{U}(u)\right\}\right]$ and

$v_{H(e)}(u)=\left[\min \left\{v_{F(a)}^{L}(u), v_{G(a)}^{L}(u)\right\}, \min \left\{v_{F(a)}^{U}(u), v_{G(a)}^{U}(u)\right\}\right]$

and is written as $(F, A) \tilde{\cap}_{E}(G, B)=(H, D)$.

\section{Example 3.9}

If we consider two fuzzy soft multi sets $(F, A)$ and $(G, B)$ as in example 3.15 , then we have (i). $(F, A) \tilde{\cup}_{E}(G, B)$

$$
\begin{aligned}
= & \left\{\left(e_{1},\left(\left\{\frac{h_{1}}{([0.4,0.5],[0.3,0.4])}, \frac{h_{2}}{([0.7,0.8][0.1,0.2])}, \frac{h_{3}}{([0.8,0.9],[0,0.1])}\right\},\left\{\frac{c_{1}}{([0.7,0.8],[0.1,0.2])}, \frac{c_{2}}{([0.6,0.7],[0.1,0.3])}\right\}\right)\right),\right. \\
& \left(e_{2},\left(\left\{\frac{h_{1}}{([0.8,0.9],[0,0.1])}, \frac{h_{2}}{([0.8,0.9],[0,0.1])}, \frac{h_{3}}{([1,1],[0,0])}\right\},\left\{\frac{c_{1}}{([0.8,0.8],[0.1,0.1])}, \frac{c_{2}}{([0.6,0.7],[0.1,0.3])}\right\}\right)\right), \\
& \left(e_{3},\left(\left\{\frac{h_{1}}{([0.7,0.8],[0.1,0.2])}, \frac{h_{2}}{([0.6,0.7],[0.1,0.3])}, \frac{h_{3}}{([1,1],[0,0])}\right\},\left\{\frac{c_{1}}{([0.2,0.3],[0.3,0.4])}, \frac{c_{2}}{([0.4,0.5][0.2,0.3])}\right\}\right)\right) \\
& \left(e_{4},\left(\left\{\frac{h_{1}}{([0.8,0.8],[0.1,0.1])}, \frac{h_{2}}{([0.2,0.3],[0.3,0.4])}, \frac{h_{3}}{([1,1],[0,0])}\right\},\left\{\frac{c_{1}}{([0.8,0.8],[0.1,0.1])}, \frac{c_{2}}{([0,7,0.8],[0.1,0.2])}\right\}\right)\right\},
\end{aligned}
$$

(ii). $(F, A) \tilde{\cap}_{E}(G, B)$

$$
\begin{aligned}
= & \left\{\left(e_{1},\left(\left\{\frac{h_{1}}{([0.2,0.3],[0.3,0.4])}, \frac{h_{2}}{([0.4,0.5][0.4,0.5])}, \frac{h_{3}}{([0.8,0.8],[0.1,0.1])}\right\},\left\{\frac{c_{1}}{([0.7,0.8],[0.1,0.2])}, \frac{c_{2}}{([0.4,0.5],[0.3,0.4])}\right\}\right),\right.\right. \\
& \left(e_{2},\left(\left\{\frac{h_{1}}{([0.4,0.5][0.2,0.5])}, \frac{h_{2}}{([0,7,0.8],[0.1,0.2])}, \frac{h_{3}}{([1,1],[0,0])}\right\},\left\{\frac{c_{1}}{([0.4,0.5][0.2,0.3])}, \frac{c_{2}}{([0.4,0.5][0.3,0.5])}\right\}\right)\right), \\
& \left(e_{3},\left(\left\{\frac{h_{1}}{([0.7,0.8],[0.1,0.2])}, \frac{h_{2}}{([0.6,0.7],[0.1,0.3])}, \frac{h_{3}}{([1,1],[0,0])}\right\},\left\{\frac{c_{1}}{([0.2,0.3],[0.3,0.4])}, \frac{c_{2}}{([0.4,0.5][0.2,0.3])}\right\}\right)\right\}, \\
& \left(e_{4},\left(\left\{\frac{h_{1}}{([0.8,0.8],[0.1,0.1])}, \frac{h_{2}}{([0.2,0.3],[0.3,0.4])}, \frac{h_{3}}{([1,1],[0,0])}\right\},\left\{\frac{c_{1}}{([0.8,0.8],[0.1,0.1])}, \frac{c_{2}}{([0,7,0.8],[0.1,0.2])}\right\}\right)\right\},
\end{aligned}
$$

\section{Proposition 3.10 (Associative Laws)}

Let $(F, A),(G, B)$ and $(H, C)$ are three interval-valued intuitionistic fuzzy soft multi sets over U, then we have the following properties:

1. $(F, A) \tilde{\cup}_{E}\left((G, B) \tilde{\cup}_{E}(H, C)\right)=\left((F, A) \tilde{\cup}_{E}(G, B)\right) \tilde{\cup}_{E}(H, C)$ 
2. $(F, A) \tilde{\cap}_{E}\left((G, B) \tilde{\cap}_{E}(H, C)\right)=\left((F, A) \tilde{\cap}_{E}(G, B)\right) \tilde{\cap}_{E}(H, C)$

Proof. In the following, we shell prove (1); (2) is proved analogously.

1. Suppose that $(G, B) \tilde{\cup}_{E}(H, C)=(I, D)$, where $D=B \cup C$ and $\forall e \in D$,

$$
I(e)= \begin{cases}G(e), & \text { if } e \in B-C \\ H(e), & \text { if } e \in C-B \\ \cup(G(e), H(e)), & \text { if } e \in B \cap C,\end{cases}
$$

Since $(F, A) \tilde{\cup}_{E}\left((G, B) \tilde{\cup}_{E}(H, C)\right)=(F, A) \tilde{\cup}_{E}(I, D)$, we suppose that $(F, A) \tilde{\cup}_{E}(I, D)=(J, M)$ where $M=A \cup D=A \cup B \cup C$ and $\forall e \in M$,

$$
J(e)= \begin{cases}G(e), & \text { if } e \in B-C-A \\ H(e), & \text { if } e \in C-B-A \\ F(e), & \text { if } e \in A-B-C \\ \cup(G(e), H(e)), & \text { if } e \in B \cap C-A, \\ \cup(F(e), H(e)), & \text { if } e \in A \cap C-B, \\ \cup(G(e), F(e)), & \text { if } e \in A \cap B-C, \\ \cup(F(e), G(e), H(e)), & \text { if } e \in A \cap B \cap C,\end{cases}
$$

Assume that $(F, A) \tilde{\cup}_{E}(G, B)=(K, S)$, where $S=A \cup B$ and $\forall e \in S$,

$$
K(e)= \begin{cases}F(e), & \text { if } e \in A-B \\ G(e), & \text { if } e \in B-A \\ \cup(F(e), G(e)), & \text { if } e \in A \cap B,\end{cases}
$$

Since $\left((F, A) \tilde{\cup}_{E}(G, B)\right) \tilde{\cup}_{E}(H, C)=(K, S) \tilde{\cup}_{E}(H, C)$, we suppose that $(K, S) \tilde{\cup}_{E}(H, C)=(L, T)$ where $T=S \cup C=A \cup B \cup C$ and $\forall e \in T$,

$$
L(e)= \begin{cases}G(e), & \text { if } e \in B-C-A \\ H(e), & \text { if } e \in C-B-A \\ F(e), & \text { if } e \in A-B-C \\ \cup(G(e), H(e)), & \text { if } e \in B \cap C-A, \\ \cup(F(e), H(e)), & \text { if } e \in A \cap C-B, \\ \cup(G(e), F(e)), & \text { if } e \in A \cap B-C, \\ \cup(F(e), G(e), H(e)), & \text { if } e \in A \cap B \cap C,\end{cases}
$$

Therefore it is clear that $M=T$ and $\forall e \in M, J(e)=L(e)$, that is $\mathrm{J}$ and $\mathrm{L}$ are the same operators. Thus $(F, A) \tilde{\cup}_{E}\left((G, B) \tilde{\cup}_{E}(H, C)\right)=\left((F, A) \tilde{\cup}_{E}(G, B)\right) \tilde{\cup}_{E}(H, C)$.

\section{Proposition 3.10 (Distributive Laws)}

Let $(F, A),(G, B)$ and $(H, C)$ are three interval-valued intuitionistic fuzzy soft multi sets over $U$, then we have the following properties:

(1). $(F, A) \tilde{\cap}_{E}\left((G, B) \tilde{\cup}_{E}(H, C)\right)=\left((F, A) \tilde{\cap}_{E}(G, B)\right) \tilde{\cup}_{E}\left((F, A) \tilde{\cap}_{E}(H, C)\right)$

(2). $(F, A) \tilde{\cup}_{E}\left((G, B) \tilde{\cap}_{E}(H, C)\right)=\left((F, A) \tilde{\cup}_{E}(G, B)\right) \tilde{\cap}_{E}\left((F, A) \tilde{\cup}_{E}(H, C)\right)$ 
Proof. In the following, we shell prove (1) and (2) is proved analogously.

(1). Suppose that $(G, B) \tilde{\cup}_{E}(H, C)=(I, D)$, where $D=B \cup C$ and $\forall e \in D$,

$$
I(e)= \begin{cases}G(e), & \text { if } e \in B-C \\ H(e), & \text { if } e \in C-B \\ \cup(G(e), H(e)), & \text { if } e \in B \cap C,\end{cases}
$$

Since $(F, A) \tilde{\cap}_{E}\left((G, B) \tilde{\cup}_{E}(H, C)\right)=(F, A) \tilde{\cap}_{E}(I, D)$, we suppose that $(F, A) \tilde{\cap}_{E}(I, D)=(J, M)$ where $M=A \cup D=A \cup B \cup C$ and $\forall e \in M$,

$$
J(e)= \begin{cases}G(e), & \text { if } e \in B-C-A \\ H(e), & \text { if } e \in C-B-A \\ F(e), & \text { if } e \in A-B-C \\ \cup(G(e), H(e)), & \text { if } e \in B \cap C-A, \\ \cap(F(e), H(e)), & \text { if } e \in A \cap C-B, \\ \cap(F(e), G(e)), & \text { if } e \in A \cap B-C, \\ \cap(F(e), U(G(e), H(e))), & \text { if } e \in A \cap B \cap C,\end{cases}
$$

Assume that $(F, A) \tilde{\cap}_{E}(G, B)=(K, S)$, where $S=A \cup B$ and $\forall e \in S$,

$$
K(e)= \begin{cases}F(e), & \text { if } e \in A-B \\ G(e), & \text { if } e \in B-A \\ \cap(F(e), G(e)), & \text { if } e \in A \cap B,\end{cases}
$$

and $(F, A) \tilde{\cap}_{E}(H, C)=(N, T)$, where $T=A \cup C$ and $\forall e \in T$,

$$
N(e)= \begin{cases}F(e), & \text { if } e \in A-C \\ H(e), & \text { if } e \in C-A \\ \cap(F(e), H(e)), & \text { if } e \in A \cap C,\end{cases}
$$

Since $\left((F, A) \tilde{\cap}_{E}(G, B)\right) \tilde{\cup}_{E}\left((F, A) \tilde{\cap}_{E}(H, C)\right)=(K, S) \tilde{\cup}_{E}(N, T)$, we suppose that $(K, S) \tilde{\cup}_{E}(N, T)=(O, P)$ where $P=S \cup T=(A \cup B) \cup(A \cup C)=A \cup B \cup C$ and $\forall e \in P$,

$$
O(e)= \begin{cases}G(e), & \text { if } e \in B-C-A \\ H(e), & \text { if } e \in C-B-A \\ F(e), & \text { if } e \in A-B-C \\ \cup(G(e), H(e)), & \text { if } e \in B \cap C-A, \\ \cap(F(e), H(e)), & \text { if } e \in A \cap C-B, \\ \cap(F(e), G(e)), & \text { if } e \in A \cap B-C, \\ \cap(F(e), \cup(G(e), H(e))), & \text { if } e \in A \cap B \cap C,\end{cases}
$$

Therefore it is clear that $M=P$ and $\forall e \in M, J(e)=O(e)$, that is " $\mathrm{J}$ " and "O" are the same operators. Thus $(F, A) \tilde{\cap}_{E}\left((G, B) \tilde{\cup}_{E}(H, C)\right)=\left((F, A) \tilde{\cap}_{E}(G, B)\right) \tilde{\cup}_{E}\left((F, A) \tilde{\cap}_{E}(H, C)\right)$.

\section{Proposition 3.11}

For two interval-valued intuitionistic fuzzy soft multi sets $(F, A)$ and $(G, B)$ over $\mathrm{U}$, then we have

1. $\left((F, A) \tilde{\cup}_{E}(G, B)\right)^{c} \subseteq(F, A)^{c} \tilde{\cup}_{E}(G, B)^{c}$

2. $(F, A)^{c} \tilde{\cap}_{E}(G, B)^{c} \tilde{\subseteq}\left((F, A) \tilde{\cap}_{E}(G, B)\right)^{c}$ 
Proof. 1. Let $(F, A) \tilde{\cup}_{E}(G, B)=(H, C)$, where $C=A \cup B$ and $\forall e \in C$,

where $U(F(e), G(e))=$

$$
H(e)= \begin{cases}F(e), & \text { if } e \in A-B \\ G(e), & \text { if } e \in B-A \\ \cup(F(e), G(e)), & \text { if } e \in A \cap B\end{cases}
$$

$$
\begin{aligned}
& \int\left\{\frac{u}{\left(\left[\max \left\{\mu_{F(a)}^{L}(u), \mu_{G(a)}^{L}(u)\right\}, \max \left\{\mu_{F(a)}^{U}(u), \mu_{G(a)}^{U}(u)\right\}\right],\left[\min \left\{v_{F(a)}^{L}(u), v_{G(a)}^{L}(u)\right\}, \min \left\{v_{F(a)}^{U}(u), v_{G(a)}^{U}(u)\right\}\right]\right)}\right. \\
& \left.\left.: u \in U_{i}\right\}: i \in I\right) .
\end{aligned}
$$

Thus $\left((F, A) \tilde{\cup}_{E}(G, B)\right)^{c}=(H, C)^{c}=\left(H^{c}, C\right)$, where $C=A \cup B$ and $\forall e \in C$,

$$
H^{c}(e)= \begin{cases}F^{c}(e), & \text { if } e \in A-B \\ G^{c}(e), & \text { if } e \in B-A \\ (\cup(F(e), G(e)))^{c}, & \text { if } e \in A \cap B,\end{cases}
$$

where $(\bigcup(F(e), G(e)))^{c}=$

$$
\begin{aligned}
& \int\left\{\frac{u}{\left(\left[\min \left\{v_{F(a)}^{L}(u), v_{G(a)}^{L}(u)\right\}, \min \left\{v_{F(a)}^{U}(u), v_{G(a)}^{U}(u)\right\}\right],\left[\max \left\{\mu_{F(a)}^{L}(u), \mu_{G(a)}^{L}(u)\right\}, \max \left\{\mu_{F(a)}^{U}(u), \mu_{G(a)}^{U}(u)\right\}\right]\right)}\right. \\
& \left.\left.: u \in U_{i}\right\}: i \in I\right) .
\end{aligned}
$$

Again, $(F, A)^{c} \tilde{\cup}_{E}(G, B)^{c}=\left(F^{c}, A\right) \tilde{\cup}_{E}\left(G^{c}, B\right)=(K, D)$. Where $D=A \cup B$ and $\forall e \in D$,

where $U\left(F^{c}(e), G^{c}(e)\right)=$

$$
K(e)= \begin{cases}F^{c}(e), & \text { if } e \in A-B \\ G^{c}(e), & \text { if } e \in B-A \\ \cup\left(F^{c}(e), G^{c}(e)\right), & \text { if } e \in A \cap B,\end{cases}
$$

$$
\int\left\{\frac{u}{\left(\left[\max \left\{v_{F(a)}^{L}(u), v_{G(a)}^{L}(u)\right\}, \max \left\{v_{F(a)}^{U}(u), \nu_{G(a)}^{U}(u)\right\}\right],\left[\min \left\{\mu_{F(a)}^{L}(u), \mu_{G(a)}^{L}(u)\right\}, \min \left\{\mu_{F(a)}^{U}(u), \mu_{G(a)}^{U}(u)\right\}\right]\right)}:\right.
$$

$\left.\left.u \in U_{i}\right\}: i \in I\right)$.

We see that $\mathrm{C}=\mathrm{D}$ and $\forall e \in C, H^{c}(e) \subseteq K(e)$. Thus $\left((F, A) \tilde{\cup}_{E}(G, B)\right)^{c} \subseteq(F, A)^{c} \tilde{\cup}_{E}(G, B)^{c}$. The other can be proved similarly.

\section{Proposition 3.12}

For two interval-valued intuitionistic fuzzy soft multi sets $(F, A)$ and $(G, B)$ over $\mathrm{U}$, then we have

$$
\begin{array}{ll}
\text { 1. } & \left((F, A) \tilde{\cup}_{R}(G, B)\right)^{c} \tilde{\subseteq}(F, A)^{c} \tilde{\cup}_{R}(G, B)^{c} \\
\text { 2. } & (F, A)^{c} \tilde{\cap}_{R}(G, B)^{c} \tilde{\subseteq}\left((F, A) \tilde{\cap}_{R}(G, B)\right)^{c} \\
\text { 3. } & \left((F, A) \tilde{\cap}_{R}(G, B)\right)^{c} \subseteq(F, A)^{c} \tilde{\cup}_{E}(G, B)^{c} \\
\text { 4. } & (F, A)^{c} \tilde{\cap}_{R}(G, B)^{c} \subseteq\left((F, A) \tilde{\cup}_{E}(G, B)\right)^{c}
\end{array}
$$

Proof. Straight forward 


\section{Proposition 3.13 (De Morgan Laws)}

For two interval-valued intuitionistic fuzzy soft multi sets $(F, A)$ and $(G, B)$ over $\mathrm{U}$, then we have

1. $\left((F, A) \tilde{\cup}_{E}(G, B)\right)^{c}=(F, A)^{c} \tilde{\cap}_{E}(G, B)^{c}$

2. $\left((F, A) \tilde{\cap}_{E}(G, B)\right)^{c}=(F, A)^{c} \tilde{\cup}_{E}(G, B)^{c}$

Proof. 1. Let $(F, A) \tilde{\cup}_{E}(G, B)=(H, C)$, where $C=A \cup B$ and $\forall e \in C$,

$$
H(e)= \begin{cases}F(e), & \text { if } e \in A-B \\ G(e), & \text { if } e \in B-A \\ \cup(F(e), G(e)), & \text { if } e \in A \cap B\end{cases}
$$

where $U(F(e), G(e))=$

$$
\int\left\{\frac{u}{\left(\left[\max \left\{\mu_{F(a)}^{L}(u), \mu_{G(a)}^{L}(u)\right\}, \max \left\{\mu_{F(a)}^{U}(u), \mu_{G(a)}^{U}(u)\right\}\right],\left[\min \left\{v_{F(a)}^{L}(u), v_{G(a)}^{L}(u)\right\}, \min \left\{v_{F(a)}^{U}(u), v_{G(a)}^{U}(u)\right\}\right]\right)}\right.
$$$$
\left.\left.: u \in U_{i}\right\}: i \in I\right) \text {. }
$$

Thus $\left((F, A) \tilde{\cup}_{E}(G, B)\right)^{c}=(H, C)^{c}=\left(H^{c}, C\right)$, where $\forall e \in C$,

$$
H^{c}(e)= \begin{cases}F^{c}(e), & \text { if } e \in A-B \\ G^{c}(e), & \text { if } e \in B-A \\ (\cup(F(e), G(e)))^{c}, & \text { if } e \in A \cap B,\end{cases}
$$

where $(\cup(F(e), G(e)))^{c}=$

$$
\begin{aligned}
& \left(\left\{\frac{u}{\left(\left[\min \left\{v_{F(a)}^{L}(u), v_{G(a)}^{L}(u)\right\}, \min \left\{v_{F(a)}^{U}(u), v_{G(a)}^{U}(u)\right\}\right], \quad\left[\max \left\{\mu_{F(a)}^{L}(u), \mu_{G(a)}^{L}(u)\right\}, \max \left\{\mu_{F(a)}^{U}(u), \mu_{G(a)}^{U}(u)\right\}\right]\right)}\right.\right. \\
& \left.\left.: u \in U_{i}\right\}: i \in I\right) .
\end{aligned}
$$

Again, $(F, A)^{c} \tilde{\cap}_{E}(G, B)^{c}=\left(F^{c}, A\right) \tilde{\cap}_{E}\left(G^{c}, B\right)=(K, D)$. Where $D=A \cup B$ and $\forall e \in D$,

where $U\left(F^{c}(e), G^{c}(e)\right)=$

$$
K(e)= \begin{cases}F^{c}(e), & \text { if } e \in A-B \\ G^{c}(e), & \text { if } e \in B-A \\ \cup\left(F^{c}(e), G^{c}(e)\right), & \text { if } e \in A \cap B,\end{cases}
$$

$$
\begin{aligned}
& \int\left\{\frac{u}{\left(\left[\min \left\{v_{F(a)}^{L}(u), v_{G(a)}^{L}(u)\right\}, \min \left\{v_{F(a)}^{U}(u), v_{G(a)}^{U}(u)\right\}\right],\left[\max \left\{\mu_{F(a)}^{L}(u), \mu_{G(a)}^{L}(u)\right\}, \max \left\{\mu_{F(a)}^{U}(u), \mu_{G(a)}^{U}(u)\right\}\right]\right)}:\right. \\
& \left.\left.u \in U_{i}\right\}: i \in I\right) .
\end{aligned}
$$

We see that $\mathrm{C}=\mathrm{D}$ and $\forall e \in C, H^{c}(e)=K(e)$. Thus $\left((F, A) \tilde{\cup}_{E}(G, B)\right)^{c}=(F, A)^{c} \tilde{\cap}_{E}(G, B)^{c}$.

The other can be proved similar

\section{Definition 3.14}

If $(\mathrm{F}, \mathrm{A})$ and $(\mathrm{G}, \mathrm{B})$ be two interval-valued intuitionistic fuzzy soft multi sets over $\mathrm{U}$, then " $(F, A)$ AND $(G, B) "$ is an interval-valued intuitionistic fuzzy soft multi set denoted by $(F, A) \wedge(G, B)$ and is defined by $(F, A) \wedge(G, B)=(H, A \times B)$, where $\mathrm{H}$ is mapping given by $\mathrm{H}$ : $\mathrm{A} \times \mathrm{B} \rightarrow \mathrm{U}$ and $\forall(a, b) \in A \times B, H(a, b)=\bigcap(F(a), G(b))=\left(\left\{\frac{u}{\left(\mu_{H(a, b)}(u), v_{H(a, b)}(u)\right)}: u \in U_{i}\right\}: i \in I\right)$, 


$$
\begin{aligned}
& \text { where } \mu_{H(a, b)}(u)=\left[\min \left\{\mu_{F(a)}^{L}(u), \mu_{G(b)}^{L}(u)\right\}, \min \left\{\mu_{F(a)}^{U}(u), \mu_{G(b)}^{U}(u)\right\}\right] \\
& \text { and } v_{H(a, b)}(u)=\left[\max \left\{v_{F(a)}^{L}(u), v_{G(b)}^{L}(u)\right\}, \max \left\{v_{F(a)}^{U}(u), v_{G(b)}^{U}(u)\right\}\right]
\end{aligned}
$$

\section{Definition 3.15}

If $(\mathrm{F}, \mathrm{A})$ and $(\mathrm{G}, \mathrm{B})$ be two interval-valued intuitionistic fuzzy soft multi sets over $\mathrm{U}$, then " $(F, A)$ OR $(G, B)$ " is an interval-valued intuitionistic fuzzy soft multi set denoted by $(F, A) \vee(G, B)$ and is defined by $(F, A) \vee(G, B)=(K, A \times B)$, where $\mathrm{K}$ is mapping given by $\mathrm{K}: \mathrm{A} \times \mathrm{B} \rightarrow \mathrm{U}$ and

$$
\begin{aligned}
& \forall(a, b) \in A \times B, K(a, b)=\bigcup(F(a), G(b))=\left(\left\{\frac{u}{\left(\mu_{K(a, b)}(u), v_{K(a, b)}(u)\right)}: u \in U_{i}\right\}: i \in I\right), \\
& \text { where } \mu_{K(a, b)}(u)=\left[\max \left\{\mu_{F(a)}^{L}(u), \mu_{G(b)}^{L}(u)\right\}, \max \left\{\mu_{F(a)}^{U}(u), \mu_{G(b)}^{U}(u)\right\}\right] \\
& \text { and } v_{K(a, b)}(u)=\left[\min \left\{v_{F(a)}^{L}(u), v_{G(b)}^{L}(u)\right\}, \min \left\{v_{F(a)}^{U}(u), v_{G(b)}^{U}(u)\right\}\right]
\end{aligned}
$$

\section{Example 3.16}

Let us consider there are two universes $U_{1}=\left\{h_{1}, h_{2}, h_{3}\right\}, U_{2}=\left\{c_{1}, c_{2}\right\}$ and let $\left\{E_{U_{1}}, E_{U_{2}}\right\}$ be a collection of sets of decision parameters related to the above universes, where $E_{U_{1}}=\left\{e_{U_{1}, 1}=\right.$ expensive, $e_{U_{1}, 2}=$ cheap, $e_{U_{1}, 3}=$ wooden $\}$ $E_{U_{1}}=\left\{e_{U_{2}, 1}=\right.$ beautiful, $e_{U_{2}, 2}=$ model, $e_{U_{2}, 3}=$ sporty $\}$,

Let $U=\prod_{i=1}^{2} F S\left(U_{i}\right), E=\prod_{i=1}^{2} E_{U_{i}}$ and

$$
A=\left\{e_{1}=\left(e_{U_{1}, 1}, e_{U_{2}, 1}\right), e_{3}=\left(e_{U_{1}, 2}, e_{U_{2}, 1}\right)\right\}, B=\left\{e_{1}=\left(e_{U_{1}, 1}, e_{U_{2}, 1}\right), e_{4}=\left(e_{U_{1}, 3}, e_{U_{2}, 2}\right)\right\} \text {. }
$$

Suppose that

$$
\begin{aligned}
&(F, A)=\{\left(e_{1},\left(\left\{\frac{h_{1}}{([0.2,0.3],[0.3,0.4])}, \frac{h_{2}}{([0.4,0.5][0.4,0.5])}, \frac{h_{3}}{([0.8,0.9],[0,0.1])}\right\},\left\{\frac{c_{1}}{([0.7,0.8],[0.1,0.2])}, \frac{c_{2}}{([0.4,0.5],[0.3,0.4])}\right\}\right)\right), \\
&\left.\left(e_{3},\left(\left\{\frac{h_{1}}{([0.7,0.8],[0.1,0.2])}, \frac{h_{2}}{([0.6,0.7],[0.1,0.3])}, \frac{h_{3}}{([1,1],[0,0])}\right\},\left\{\frac{c_{1}}{([0.2,0.3],[0.3,0.4])}, \frac{c_{2}}{([0.4,0.5][0.2,0.3])}\right\}\right)\right)\right\}, \\
&(G, B)=\left\{\left(e_{1},\left(\left\{\frac{h_{1}}{([0.4,0.5][0.3,0.4])}, \frac{h_{2}}{([0,7,0.8],[0.1,0.2])}, \frac{h_{3}}{([0.8,0.8],[0.1,0.1])}\right\},\left\{\frac{c_{1}}{([0,7,0.8],[0.1,0.2])}, \frac{c_{2}}{([0.6,0.7],[0.1,0.3])}\right\}\right)\right\},\right. \\
&\left.\left(e_{4},\left(\left\{\frac{h_{1}}{([0.8,0.8],[0.1,0.1])}, \frac{h_{2}}{([0.2,0.3],[0.3,0.4])}, \frac{h_{3}}{([1,1],[0,0])}\right\},\left\{\frac{c_{1}}{([0.8,0.8],[0.1,0.1])}, \frac{c_{2}}{([0,7,0.8],[0.1,0.2])}\right\}\right)\right)\right\},
\end{aligned}
$$

be two interval-valued intuitionistic fuzzy soft multi sets over $\mathrm{U}$, then we have,

(1). $(F, A) \wedge(G, B)$

$$
\begin{aligned}
= & \left\{\left(e_{1}, e_{1}\right),\left(\left\{\frac{h_{1}}{([0.2,0.3],[0.3,0.4])}, \frac{h_{2}}{([0.4,0.5][0.4,0.5])}, \frac{h_{3}}{([0.8,0.8],[0.1,0.1])}\right\},\left\{\frac{c_{1}}{([0.7,0.8],[0.1,0.2])}, \frac{c_{2}}{([0.4,0.5],[0.3,0.4])}\right\}\right)\right), \\
& \left(\left(e_{1}, e_{4}\right),\left(\left\{\frac{h_{1}}{([0.2,0.3],[0.3,0.4])}, \frac{h_{2}}{([0.2,0.3][0.4,0.5])}, \frac{h_{3}}{([0.8,0.9],[0,0.1])}\right\},\left\{\frac{c_{1}}{([0.7,0.8],[0.1,0.2])}, \frac{c_{2}}{([0.4,0.5],[0.3,0.4])}\right\}\right)\right), \\
& \left(\left(e_{3}, e_{1}\right),\left(\left\{\frac{h_{1}}{([0.4,0.5][0.3,0.4])}, \frac{h_{2}}{([0.6,0.7],[0.1,0.3])}, \frac{h_{3}}{([0.8,0.8],[0.1,0.1])}\right\},\left\{\frac{c_{1}}{([0.2,0.3],[0.3,0.4])}, \frac{c_{2}}{([0.4,0.5][0.2,0.3])}\right\}\right)\right), \\
& \left.\left(\left(e_{3}, e_{4}\right),\left(\left\{\frac{h_{1}}{([0.7,0.8],[0.1,0.2])}, \frac{h_{2}}{([0.2,0.3],[0.3,0.4])}, \frac{h_{3}}{([1,1],[0,0])}\right\},\left\{\frac{c_{1}}{([0.2,0.3],[0.3,0.4])}, \frac{c_{2}}{([0.4,0.5][0.2,0.3])}\right\}\right)\right)\right\}
\end{aligned}
$$


Advances in Vision Computing: An International Journal (AVC) Vol.2, No.2, June 2015

(2). $(F, A) \vee(G, B)$

$$
\begin{aligned}
= & \left\{\left(\left(e_{1}, e_{1}\right),\left(\left\{\frac{h_{1}}{([0.4,0.5][0.3,0.4])}, \frac{h_{2}}{([0,7,0.8],[0.1,0.2])}, \frac{h_{3}}{([0.8,0.9],[0,0.1])}\right\},\left\{\frac{c_{1}}{([0,7,0.8],[0.1,0.2])}, \frac{c_{2}}{([0.6,0.7],[0.1,0.3])}\right\}\right)\right),\right. \\
& \left(\left(e_{1}, e_{4}\right),\left(\left\{\frac{h_{1}}{([0.8,0.8],[0.1,0.1])}, \frac{h_{2}}{([0.4,0.5][0.4,0.5])}, \frac{h_{3}}{([1,1],[0,0])}\right\},\left\{\frac{c_{1}}{([0.8,0.8],[0.1,0.1])}, \frac{c_{2}}{([0,7,0.8],[0.1,0.2])}\right\}\right)\right), \\
& \left(\left(e_{3}, e_{1}\right),\left(\left\{\frac{h_{1}}{([0.7,0.8],[0.1,0.2])}, \frac{h_{2}}{([0,7,0.8],[0.1,0.2])}, \frac{h_{3}}{([1,1],[0,0])}\right\},\left\{\frac{c_{1}}{([0,7,0.8],[0.1,0.2])}, \frac{c_{2}}{([0.6,0.7],[0.1,0.3])}\right\}\right)\right), \\
& \left.\left(\left(e_{3}, e_{4}\right),\left(\left\{\frac{h_{1}}{([0.8,0.8],[0.1,0.1])}, \frac{h_{2}}{([0.6,0.7],[0.1,0.3])}, \frac{h_{3}}{([1,1],[0,0])}\right\},\left\{\frac{c_{1}}{([0.8,0.8],[0.1,0.1])}, \frac{c_{2}}{([0,7,0.8],[0.1,0.2])}\right\}\right)\right)\right\}
\end{aligned}
$$

\section{Proposition 3.17}

For two interval-valued intuitionistic fuzzy soft multi sets $(F, A)$ and $(G, B)$ over $U$, we have the following

$$
\begin{aligned}
& \text { 1. }((F, A) \wedge(G, B))^{c}=(F, A)^{c} \vee(G, B)^{c} \\
& \text { 2. }((F, A) \vee(G, B))^{c}=(F, A)^{c} \wedge(G, B)^{c}
\end{aligned}
$$

Proof. 1. Let $(F, A) \wedge(G, B)=(H, A \times B)$, where $\forall a \in A$ and $\forall b \in B$,

$$
\begin{aligned}
& H(a, b)=\bigcap(F(a), G(b))=\left(\left\{\frac{u}{\left(\mu_{H(a, b)}(u), v_{H(a, b)}(u)\right)}: u \in U_{i}\right\}: i \in I\right) \\
& \mu_{H(a, b)}(u)=\left[\min \left\{\mu_{F(a)}^{L}(u), \mu_{G(b)}^{L}(u)\right\}, \min \left\{\mu_{F(a)}^{U}(u), \mu_{G(b)}^{U}(u)\right\}\right], \\
& v_{H(a, b)}(u)=\left[\max \left\{v_{F(a)}^{L}(u), v_{G(b)}^{L}(u)\right\}, \max \left\{v_{F(a)}^{U}(u), v_{G(b)}^{U}(u)\right\}\right]
\end{aligned}
$$

Thus $((F, A) \wedge(G, B))^{c}=(H, A \times B)^{c}=\left(H^{c}, A \times B\right)$, where $\forall(a, b) \in A \times B$,

$$
\begin{aligned}
& H^{c}(a, b)=(H(a, b))^{c}= \\
& \left(\left\{\frac{u}{\left(\left[\max \left\{v_{F(a)}^{L}(u), v_{G(a)}^{L}(u)\right\}, \max \left\{v_{F(a)}^{U}(u), v_{G(a)}^{U}(u)\right\}\right],\left[\min \left\{\mu_{F(a)}^{L}(u), \mu_{G(a)}^{L}(u)\right\}, \min \left\{\mu_{F(a)}^{U}(u), \mu_{G(a)}^{U}(u)\right\}\right]\right)}:\right.\right. \\
& \left.\left.u \in U_{i}\right\}: i \in I\right) .
\end{aligned}
$$

Again, let $(F, A)^{c} \vee(G, B)^{c}=\left(F^{c}, A\right) \vee\left(G^{c}, B\right)=(K, A \times B)$, where $\forall(a, b) \in A \times B$,

$$
\begin{aligned}
& K(a, b)=\bigcup\left(F^{c}(a), G^{c}(b)\right)= \\
& \left(\left\{\frac{u}{\left(\left[\max \left\{v_{F(a)}^{L}(u), v_{G(a)}^{L}(u)\right\}, \max \left\{v_{F(a)}^{U}(u), v_{G(a)}^{U}(u)\right\}\right],\left[\min \left\{\mu_{F(a)}^{L}(u), \mu_{G(a)}^{L}(u)\right\}, \min \left\{\mu_{F(a)}^{U}(u), \mu_{G(a)}^{U}(u)\right\}\right]\right)}:\right.\right. \\
& \left.\left.u \in U_{i}\right\}: i \in I\right) .
\end{aligned}
$$

Thus it follows that $((F, A) \wedge(G, B))^{c}=(F, A)^{c} \vee(G, B)^{c}$.

The other can be proved similarly.

\section{Proposition 3.18 (Associative Laws)}

Let $(F, A),(G, B)$ and $(H, C)$ are three interval valued intuitionistic fuzzy soft multi sets over $U$, then we have the following properties:

1. $(F, A) \wedge((G, B) \wedge(H, C))=((F, A) \wedge(G, B)) \wedge(H, C)$ 
2. $(F, A) \vee((G, B) \vee(H, C))=((F, A) \vee(G, B)) \vee(H, C)$

Proof. 1. Assume that $(G, B) \wedge(H, C)=(I, B \times C)$, where $\forall(b, c) \in B \times C, I(b, c)=G(b) \cap H(c)$

$$
\begin{aligned}
=\left(\left\{\frac{u}{\left(\mu_{I(b, c)}(u), v_{I(b, c)}(u)\right)}: u\right.\right. & \left.\left.\in U_{i}\right\}: i \in I\right) \\
\mu_{I(b, c)}(u) & =\left[\min \left\{\mu_{G(b)}^{L}(u), \mu_{H(c)}^{L}(u)\right\}, \min \left\{\mu_{G(b)}^{U}(u), \mu_{H(c)}^{U}(u)\right\}\right], \\
V_{I(b, c)}(u) & =\left[\max \left\{v_{G(b)}^{L}(u), v_{H(c)}^{L}(u)\right\}, \max \left\{v_{G(b)}^{U}(u), v_{H(c)}^{U}(u)\right\}\right]
\end{aligned}
$$

Since $(F, A) \wedge((G, B) \wedge(H, C))=(F, A) \wedge(I, B \times C)$, we suppose that $(F, A) \wedge(I, B \times C)=(K, A \times(B \times C))$ where

$$
\begin{aligned}
\forall(a, b, c) \in A \times( & B \times C)=A \times B \times C, K(a, b, c)=F(a) \cap I(b, c)=\left(\left\{\frac{u}{\left(\mu_{K(a, b, c)}(u), v_{K(a, b, c)}(u)\right)}: u \in U_{i}\right\}: i \in I\right) \\
& \mu_{K(a, b, c)}(u)=\left[\min \left\{\mu_{F(a)}^{L}(u), \mu_{I(b, c)}^{L}(u)\right\}, \min \left\{\mu_{F(a)}^{U}(u), \mu_{I(b, c)}^{U}(u)\right\}\right] \\
& =\left[\min \left\{\mu_{F(a)}^{L}(u), \min \left\{\mu_{G(b)}^{L}(u), \mu_{H(c)}^{L}(u)\right\}\right\}, \min \left\{\mu_{F(a)}^{U}(u), \min \left\{\mu_{G(b)}^{U}(u), \mu_{H(c)}^{U}(u)\right\}\right\}\right] \\
& =\left[\min \left\{\mu_{F(a)}^{L}(u), \mu_{G(b)}^{L}(u), \mu_{H(c)}^{L}(u)\right\}, \min \left\{\mu_{F(a)}^{U}(u), \mu_{G(b)}^{U}(u), \mu_{H(c)}^{U}(u)\right\}\right] \\
& V_{K(a, b, c)}(u)=\left[\max \left\{v_{F(a)}^{L}(u), v_{I(b, c)}^{L}(u)\right\}, \max \left\{v_{F(a)}^{U}(u), v_{I(b, c)}^{U}(u)\right\}\right] \\
& =\left[\max \left\{v_{F(a)}^{L}(u), \max \left\{v_{G(b)}^{L}(u), v_{H(c)}^{L}(u)\right\}\right\}, \max \left\{v_{F(a)}^{U}(u), \max \left\{v_{G(b)}^{U}(u), v_{H(c)}^{U}(u)\right\}\right\}\right] \\
& =\left[\max \left\{v_{F(a)}^{L}(u), v_{G(b)}^{L}(u), v_{H(c)}^{L}(u)\right\}, \max \left\{v_{F(a)}^{U}(u), v_{G(b)}^{U}(u), v_{H(c)}^{U}(u)\right\}\right] .
\end{aligned}
$$

We take $(a, b) \in A \times B$. Suppose that $(F, A) \wedge(G, B)=(J, A \times B)$ where $\forall(a, b) \in A \times B$,

$$
\begin{aligned}
J(a, b)=F(a) \cap G(b)= & \left(\left\{\frac{u}{\left(\mu_{J(a, b)}(u), v_{J(a, b)}(u)\right)}: u \in U_{i}\right\}: i \in I\right) \\
& \mu_{J(a, b)}(u)=\left[\min \left\{\mu_{F(a)}^{L}(u), \mu_{G(b)}^{L}(u)\right\}, \min \left\{\mu_{F(a)}^{U}(u), \mu_{G(b)}^{U}(u)\right\}\right], \\
& v_{J(a, b)}(u)=\left[\max \left\{v_{F(a)}^{L}(u), v_{G(b)}^{L}(u)\right\}, \max \left\{v_{F(a)}^{U}(u), v_{G(b)}^{U}(u)\right\}\right]
\end{aligned}
$$

Since $((F, A) \wedge(G, B)) \wedge(H, C)=(J, A \times B) \wedge(H, C)$, we suppose that $(J, A \times B) \wedge(H, C)=(O,(A \times B) \times C)$ where $\forall(a, b, c) \in(A \times B) \times C=A \times B \times C$,

$$
\begin{gathered}
O(a, b, c)=J(a, b) \cap H(c)=\left(\left\{\frac{u}{\left(\mu_{O(a, b, c)}(u), v_{O(a, b, c)}(u)\right)}: u \in U_{i}\right\}: i \in I\right) \\
\mu_{O(a, b, c)}(u)=\left[\min \left\{\mu_{J(a, b)}^{L}(u), \mu_{H(c)}^{L}(u)\right\}, \min \left\{\mu_{J(a, b)}^{U}(u), \mu_{H(c)}^{U}(u)\right\}\right] \\
=\left[\min \left\{\min \left\{\mu_{F(a)}^{L}(u), \mu_{G(b)}^{L}(u)\right\}, \mu_{H(c)}^{L}(u)\right\}, \min \left\{\min \left\{\mu_{F(a)}^{U}(u), \mu_{G(b)}^{U}(u)\right\}, \mu_{H(c)}^{U}(u)\right\}\right] \\
=\left[\min \left\{\mu_{F(a)}^{L}(u), \mu_{G(b)}^{L}(u), \mu_{H(c)}^{L}(u)\right\}, \min \left\{\mu_{F(a)}^{U}(u), \mu_{G(b)}^{U}(u), \mu_{H(c)}^{U}(u)\right\}\right]=\mu_{K(a, b, c)}(u)
\end{gathered}
$$

and

$$
\begin{aligned}
& v_{O(a, b, c)}(u)=\left[\max \left\{v_{J(a, b)}^{L}(u), v_{H(c)}^{L}(u)\right\}, \max \left\{v_{J(a, b)}^{U}(u), v_{H(c)}^{U}(u)\right\}\right] \\
& =\left[\max \left\{\max \left\{v_{F(a)}^{L}(u), v_{G(b)}^{L}(u)\right\}, v_{H(c)}^{L}(u)\right\}, \max \left\{\max \left\{v_{F(a)}^{U}(u), v_{G(b)}^{U}(u)\right\}, v_{H(c)}^{U}(u)\right\}\right] \\
& =\left[\max \left\{v_{F(a)}^{L}(u), v_{G(b)}^{L}(u), v_{H(c)}^{L}(u)\right\}, \max \left\{v_{F(a)}^{U}(u), v_{G(b)}^{U}(u), v_{H(c)}^{U}(u)\right\}\right]=v_{K(a, b, c)}(u)
\end{aligned}
$$

Consequently, $\mathrm{K}$ and $\mathrm{O}$ are the same operators. Thus

$(F, A) \wedge((G, B) \wedge(H, C))=((F, A) \wedge(G, B)) \wedge(H, C)$.

The proof of (2) is similar to that of (1). 


\section{Definition 3.19}

The necessity operation on an interval-valued intuitionistic fuzzy multi set $(\mathrm{F}, \mathrm{A})$ is denoted by $\square(F, A)$ and is defined as $\square(F, A)=(\square F, A)$, where $\forall a \in A$

$$
\square F(a)=\left\{\left\{\frac{u}{\left(\mu_{\square F(a)}(u), v_{\square F(a)}(u)\right)}: u \in U_{i}\right\}: i \in I\right) .
$$

Here $\mu_{\square F(a)}(u)=\left[\mu_{F(a)}^{L}(u), \mu_{F(a)}^{U}(u)\right]$ is the interval-valued fuzzy membership degree of the object $u \in U_{i}$ holds on parameter $a \in A, \quad v_{\square F(a)}(u)=\left[1-\mu_{F(a)}^{U}(u), 1-\mu_{F(a)}^{L}(u)\right]$ is the interval-valued fuzzy membership degree of the object $u \in U_{i}$ does not hold on parameter $a \in A$.

\section{Proposition 3.20}

Let $(\mathrm{F}, \mathrm{A})$ and $(\mathrm{G}, \mathrm{B})$ are two interval-valued intuitionistic fuzzy soft multi sets over $\mathrm{U}$, then we have the following properties:

1. $\square\left((F, A) \tilde{\cup}_{R}(G, B)\right)=\square(F, A) \tilde{\cup}_{R} \square(G, B)$

2. $\square\left((F, A) \tilde{\cap}_{R}(G, B)\right)=\square(F, A) \tilde{\cap}_{R} \square(G, B)$

3. $\square\left((F, A) \tilde{\cup}_{E}(G, B)\right)=\square(F, A) \tilde{\cup}_{E}^{\square(G, B)}$

4. $\square\left((F, A) \tilde{\cap}_{E}(G, B)\right)=\square(F, A) \tilde{\cap}_{E} \square(G, B)$

5. $\square((F, A) \wedge(G, B))=\square(F, A) \wedge \square(G, B)$

6. $\square((F, A) \vee(G, B))=\square(F, A) \vee \square(G, B)$

7. $\square \square(F, A)=\square(F, A)$

Proof. 1. Assume that $(F, A) \tilde{\cup}_{R}(G, B)=(H, C)$, where $C=A \cap B$ and $\forall e \in C$,

$$
\begin{aligned}
& H(e)=\cup(F(e), G(e))= \\
& \left\{\left\{\frac{u}{\left(\left[\max \left\{\mu_{F(e)}^{L}(u), \mu_{G(e)}^{L}(u)\right\}, \max \left\{\mu_{F(e)}^{U}(u), \mu_{G(e)}^{U}(u)\right\}\right],\left[\min \left\{v_{F(e)}^{L}(u), v_{G(e)}^{L}(u)\right\}, \min \left\{v_{F(e)}^{U}(u), v_{G(e)}^{U}(u)\right\}\right]\right)}:\right.\right. \\
& \left.\left.u \in U_{i}\right\}: i \in I\right)
\end{aligned}
$$

Thus $\square\left((F, A) \tilde{\cup}_{R}(G, B)\right)=\square(H, C)=(\square H, C)$, where $\forall e \in C$

$$
\square H(e)=\left(\left\{\frac{u}{\left(\mu_{\square H(e)}(u), v_{\square H(e)}(u)\right)}: u \in U_{i}\right\}: i \in I\right),
$$

where $\mu_{\square H(e)}(u)=\left[\max \left\{\mu_{F(e)}^{L}(u), \mu_{G(e)}^{L}(u)\right\}, \max \left\{\mu_{F(e)}^{U}(u), \mu_{G(e)}^{U}(u)\right\}\right]$

$$
\text { and } v_{\square H(e)}(u)=\left[1-\max \left\{\mu_{F(e)}^{U}(u), \mu_{G(e)}^{U}(u)\right\}, 1-\max \left\{\mu_{F(e)}^{L}(u), \mu_{G(e)}^{L}(u)\right\}\right]
$$

Also $\square(F, A)=(\square F, A)$, where $\forall e \in A$

$$
\square F(e)=\left(\left\{\frac{u}{\left(\left[\mu_{F(e)}^{L}(u), \mu_{F(e)}^{U}(u)\right],\left[1-\mu_{F(e)}^{U}(u), 1-\mu_{F(e)}^{L}(u)\right]\right)}: u \in U_{i}\right\}: i \in I\right)
$$

and $\square(G, B)=(\square G, B)$, where $\forall e \in B$

$$
\square G(e)=\left(\left\{\frac{u}{\left(\left[\mu_{G(e)}^{L}(u), \mu_{G(e)}^{U}(u)\right],\left[1-\mu_{G(e)}^{U}(u), 1-\mu_{G(e)}^{L}(u)\right]\right)}: u \in U_{i}\right\}: i \in I\right)
$$


Again, let $\square(F, A) \tilde{\cup}_{R} \square(G, B)=(\square F, A) \tilde{\cup}_{R}(\square G, B)=(K, C)$, where $C=A \cap B$ and $\forall e \in C$,

$$
\begin{aligned}
K(e) & =\bigcup(\square F(e), \square G(e))=\left(\left\{\frac{u}{\left(\mu_{K(e)}(u), v_{K(e)}(u)\right)}: u \in U_{i}\right\}: i \in I\right), \\
\text { where } \mu_{K(e)}(u)= & {\left[\max \left\{\mu_{F(e)}^{L}(u), \mu_{G(e)}^{L}(u)\right\}, \max \left\{\mu_{F(e)}^{U}(u), \mu_{G(e)}^{U}(u)\right\}\right]=\mu_{\square H(e)}(u) } \\
\text { and } v_{K(e)}(u)= & {\left[\min \left\{1-\mu_{F(e)}^{U}(u), 1-\mu_{G(e)}^{U}(u)\right\}, \min \left\{1-\mu_{F(e)}^{L}(u), 1-\mu_{G(e)}^{L}(u)\right\}\right] } \\
= & {\left[1-\max \left\{\mu_{F(e)}^{U}(u), \mu_{G(e)}^{U}(u)\right\}, 1-\max \left\{\mu_{F(e)}^{L}(u), \mu_{G(e)}^{L}(u)\right\}\right]=v_{\square H(e)}(u) }
\end{aligned}
$$

Consequently, $\square(H, C)$ and $(K, C)$ are the same interval-valued intuitionistic fuzzy soft multi sets. Thus $\square\left((F, A) \tilde{\cup}_{R}(G, B)\right)=\square(F, A) \tilde{\cup}_{R} \square(G, B)$.

The proofs of (2)-(7) are similar to that of (1).

\section{Definition 3.21}

The possibility operation on an interval-valued intuitionistic fuzzy multi set $(\mathrm{F}, \mathrm{A})$ is denoted by $\Delta(F, A)$ and is defined as $\Delta(F, A)=(\Delta F, A)$, where $\forall a \in A$

$$
\Delta F(a)=\left(\left\{\frac{u}{\left(\mu_{\Delta F(a)}(u), v_{\Delta F(a)}(u)\right)}: u \in U_{i}\right\}: i \in I\right) .
$$

Here $v_{\Delta F(a)}(u)=\left[v_{\Delta F(a)}^{L}(u), v_{\Delta F(a)}^{U}(u)\right]$ is the interval-valued fuzzy membership degree of the object $u \in U_{i}$ does not hold on parameter $a \in A, \mu_{\Delta F(a)}(u)=\left[1-v_{\Delta F(a)}^{U}(u), 1-v_{\Delta F(a)}^{L}(u)\right]$ is the intervalvalued fuzzy membership degree of the object $u \in U_{i}$ holds on parameter $a \in A$.

\section{Proposition 3.22}

Let (F, A) and (G, B) are two interval-valued intuitionistic fuzzy soft multi sets over $\mathrm{U}$, then we have the following properties:

1. $\Delta\left((F, A) \tilde{\cup}_{R}(G, B)\right)=\Delta(F, A) \tilde{\cup}_{R} \Delta(G, B)$

2. $\Delta\left((F, A) \tilde{\cap}_{R}(G, B)\right)=\Delta(F, A) \tilde{\cap}_{R} \Delta(G, B)$

3. $\Delta\left((F, A) \tilde{\cup}_{E}(G, B)\right)=\Delta(F, A) \tilde{\cup}_{E} \Delta(G, B)$

4. $\Delta\left((F, A) \tilde{\cap}_{E}(G, B)\right)=\Delta(F, A) \tilde{\cap}_{E} \Delta(G, B)$

5. $\Delta((F, A) \wedge(G, B))=\Delta(F, A) \wedge \Delta(G, B)$

6. $\Delta((F, A) \vee(G, B))=\Delta(F, A) \vee \Delta(G, B)$

7. $\Delta \Delta(F, A)=\Delta(F, A)$

Proof. 1. Assume that $(F, A) \tilde{\cup}_{R}(G, B)=(H, C)$, where $C=A \cap B$ and $\forall e \in C$,

$$
\begin{aligned}
& H(e)=\bigcup(F(e), G(e))= \\
& \left(\left\{\frac{u}{\left(\left[\max \left\{\mu_{F(e)}^{L}(u), \mu_{G(e)}^{L}(u)\right\}, \max \left\{\mu_{F(e)}^{U}(u), \mu_{G(e)}^{U}(u)\right\}\right],\left[\min \left\{v_{F(e)}^{L}(u), v_{G(e)}^{L}(u)\right\}, \min \left\{v_{F(e)}^{U}(u), v_{G(e)}^{U}(u)\right\}\right]\right)}:\right.\right. \\
& \left.\left.u \in U_{i}\right\}: i \in I\right)
\end{aligned}
$$

Thus $\Delta\left((F, A) \tilde{\cup}_{R}(G, B)\right)=\Delta(H, C)=(\Delta H, C)$, where $\forall e \in C$

$$
\Delta H(e)=\left(\left\{\frac{u}{\left(\mu_{\Delta H(e)}(u), v_{\Delta H(e)}(u)\right)}: u \in U_{i}\right\}: i \in I\right),
$$


where $\mu_{\Delta H(e)}(u)=\left[1-\min \left\{v_{F(e)}^{U}(u), v_{G(e)}^{U}(u)\right\}, 1-\min \left\{v_{F(e)}^{L}(u), v_{G(e)}^{L}(u)\right\}\right]$

and $v_{\Delta H(e)}(u)=\left[\min \left\{v_{F(e)}^{L}(u), v_{G(e)}^{L}(u)\right\}, \min \left\{v_{F(e)}^{U}(u), v_{G(e)}^{U}(u)\right\}\right]$

Also $\Delta(F, A)=(\Delta F, A)$, where $\forall e \in A$

$$
\Delta F(e)=\left(\left\{\frac{u}{\left(\left[1-v_{F(e)}^{U}(u), 1-v_{F(e)}^{L}(u)\right],\left[v_{F(e)}^{L}(u), v_{F(e)}^{U}(u)\right]\right)}: u \in U_{i}\right\}: i \in I\right)
$$

and $\Delta(G, B)=(\Delta G, B)$, where $\forall e \in B$

$$
\Delta G(e)=\left(\left\{\frac{u}{\left(\left[1-v_{G(e)}^{U}(u), 1-v_{G(e)}^{L}(u)\right],\left[v_{G(e)}^{L}(u), v_{G(e)}^{U}(u)\right]\right)}: u \in U_{i}\right\}: i \in I\right)
$$

Again, let $\Delta(F, A) \tilde{\cup}_{R} \Delta(G, B)=(\Delta F, A) \tilde{\cup}_{R}(\Delta G, B)=(K, C)$, where $C=A \cap B$ and $\forall e \in C$,

$$
K(e)=\bigcup(\Delta F(e), \Delta G(e))=\left(\left\{\frac{u}{\left(\mu_{K(e)}(u), v_{K(e)}(u)\right)}: u \in U_{i}\right\}: i \in I\right),
$$

where $\mu_{K(e)}(u)=\left[\max \left\{1-v_{F(e)}^{U}(u), 1-v_{G(e)}^{U}(u)\right\}, \max \left\{1-v_{F(e)}^{L}(u), 1-v_{G(e)}^{L}(u)\right\}\right]$

$$
=\left[1-\min \left\{\mu_{F(e)}^{U}(u), \mu_{G(e)}^{U}(u)\right\}, 1-\min \left\{\mu_{F(e)}^{L}(u), \mu_{G(e)}^{L}(u)\right\}\right]=\mu_{\Delta H(e)}(u)
$$

and $v_{K(e)}(u)=\left[\min \left\{v_{F(e)}^{L}(u), v_{G(e)}^{L}(u)\right\}, \min \left\{v_{F(e)}^{U}(u), v_{G(e)}^{U}(u)\right\}\right]=v_{\Delta H(e)}(u)$

Consequently, $\Delta(H, C)$ and $(K, C)$ are the same interval-valued intuitionistic fuzzy soft multi sets. Thus $\Delta\left((F, A) \tilde{\cup}_{R}(G, B)\right)=\Delta(F, A) \tilde{\cup}_{R} \Delta(G, B)$.

The proofs of (2)-(7) are similar to that of (1).

\section{Proposition 3.23}

Let $(F, A)$ be any interval-valued intuitionistic fuzzy soft multi set over $\mathrm{U}$, then we have the following properties:

1. $\square(F, A) \tilde{\subseteq}(F, A) \tilde{\subseteq} \Delta(F, A)$

2. $\Delta \square(F, A)=\square(F, A)$

3. $\square \Delta(F, A)=\Delta(F, A)$

Proof. Assume that $(\mathrm{F}, \mathrm{A})$ be any interval-valued intuitionistic fuzzy soft multi set over $\mathrm{U}$, such that $\forall e \in A$,

$$
F(e)=\left(\left\{\frac{u}{\left(\left[\mu_{F(e)}^{L}(u), \mu_{F(e)}^{U}(u)\right],\left[v_{F(e)}^{L}(u), v_{F(e)}^{U}(u)\right]\right)}: u \in U_{i}\right\}: i \in I\right)
$$

Then, $\square(F, A)=(\square F, A)$, where $\forall e \in A$

$$
\square F(e)=\left(\left\{\frac{u}{\left(\left[\mu_{F(e)}^{L}(u), \mu_{F(e)}^{U}(u)\right],\left[1-\mu_{F(e)}^{U}(u), 1-\mu_{F(e)}^{L}(u)\right]\right)}: u \in U_{i}\right\}: i \in I\right)
$$

and $\Delta(F, A)=(\Delta F, A)$, where $\forall e \in A$

$$
\Delta F(e)=\left(\left\{\frac{u}{\left(\left[1-v_{F(e)}^{U}(u), 1-v_{F(e)}^{L}(u)\right],\left[v_{F(e)}^{L}(u), v_{F(e)}^{U}(u)\right]\right)}: u \in U_{i}\right\}: i \in I\right) .
$$


1. Since $\mu_{F(e)}^{U}(u)+v_{F(e)}^{U}(u) \leq 1$, implies that $1-\mu_{F(e)}^{U}(u) \geq v_{F(e)}^{U}(u)$.

Hence $\quad 1-\mu_{F(e)}^{U}(u) \geq v_{F(e)}^{U}(u) \geq v_{F(e)}^{L}(u) \quad$ and $\quad 1-\mu_{F(e)}^{L}(u) \geq 1-\mu_{F(e)}^{U}(u) \geq v_{F(e)}^{U}(u)$. Thus $\square(F, A) \tilde{\subseteq}(F, A)$.

Again since $\mu_{F(e)}^{U}(u)+v_{F(e)}^{U}(u) \leq 1$, implies that $1-v_{F(e)}^{U}(u) \geq \mu_{F(e)}^{U}(u)$. Hence $1-v_{F(e)}^{U}(u) \geq \mu_{F(e)}^{U}(u) \geq \mu_{F(e)}^{L}(u) \quad$ and $\quad 1-v_{F(e)}^{L}(u) \geq 1-v_{F(e)}^{U}(u) \geq \mu_{F(e)}^{U}(u)$. Thus $(F, A) \tilde{\subseteq} \Delta(F, A)$.

Consequently, $\square(F, A) \tilde{\subseteq}(F, A) \tilde{\subseteq} \Delta(F, A)$.

2. We have, $\square(F, A)=(\square F, A)$, where $\forall e \in A$

$$
\square F(e)=\left(\left\{\frac{u}{\left(\left[\mu_{F(e)}^{L}(u), \mu_{F(e)}^{U}(u)\right],\left[1-\mu_{F(e)}^{U}(u), 1-\mu_{F(e)}^{L}(u)\right]\right)}: u \in U_{i}\right\}: i \in I\right)
$$

Therefore, $\Delta \square(F, A)=(\Delta \square F, A)$, where $\forall e \in A$

$$
\begin{aligned}
& \Delta \square F(e)=\left(\left\{\frac{u}{\left(\left[1-\left(1-\mu_{F(e)}^{L}(u)\right), 1-\left(1-\mu_{F(e)}^{U}(u)\right)\right],\left[1-\mu_{F(e)}^{U}(u), 1-\mu_{F(e)}^{L}(u)\right]\right)}: u \in U_{i}\right\}: i \in I\right) \\
& =\left(\left\{\frac{u}{\left(\left[\mu_{F(e)}^{L}(u), \mu_{F(e)}^{U}(u)\right],\left[1-\mu_{F(e)}^{U}(u), 1-\mu_{F(e)}^{L}(u)\right]\right)}: u \in U_{i}\right\}: i \in I\right)=\square F(e)
\end{aligned}
$$

Consequently, $\Delta \square(F, A)=\square(F, A)$

The proof of (3) is similar to that of (2).

\section{CONCLUSIONS}

In this study, we define some operations in interval-valued intuitionistic fuzzy soft multi set theory and show that the associative, distribution and De Morgan's type of results hold in interval-valued intuitionistic fuzzy soft multi set theory for the newly defined operations in our way. Also, we define the necessity and possibility operations on interval-valued intuitionistic fuzzy soft multi set theory and study their basic properties and some results.

\section{REFERENCES}

[1] K.Alhazaymeh \& N.Hassan,(2014) "Vague Soft Multiset Theory", Int. J. Pure and Applied Math., Vol. 93, pp511-523.

[2] M.I.Ali,F.Feng,X. Liu, W.K. Minc \& M. Shabir, (2009) "On some new operations in soft set theory", Comp. Math. Appl., vol. 57, pp1547-1553.

[3] S.Alkhazaleh \& A.R. Salleh, (2012) "Fuzzy Soft Multi sets Theory", Hindawi Publishing Corporation, Abstract and Applied Analysis, Article ID 350603, 20 pages, doi: 10.1155/2012/350603.

[4] S.Alkhazaleh,A.R.Salleh \& N. Hassan, (2011) Soft Multi sets Theory, Appl. Math. Sci., Vol. 5, pp3561-3573.

[5] K.Atanassov,(1986) "Intuitionistic fuzzy sets", Fuzzy Sets and Systems, Vol. 20, pp87-96.

[6] K.Atanassov \& G.Gargov,(1989) "Interval valued intuitionistic fuzzy sets, Fuzzy Sets and Systems, Vol. 31, pp343-349.

[7] K.VBabitha \& S.J.John,(2013) “On Soft Multi sets”, Ann. Fuzzy Math. Inform., Vol. 5 pp35-44.

[8] H.M.Balami \& A. M.Ibrahim,(2013) "Soft Multiset and its Application in Information System", International Journal of scientific research and management, Vol. 1, pp471-482. 
[9] T.M.Basu, N.K. Mahapatra \& S.K. Mondal, (2012) "On some new operations in fuzzy soft set and intuitionistic fuzzy soft set theory”, Int. J. Comp. Sci. Inform. Technology and Security., Vol. 2, pp408-417.

[10] M.Bora,T.J.Nego \& D.K. Sut, (2012) "Some results on intuitionistic fuzzy soft sets", Int. J. Math. Trends and Technology, Vol. 3, pp63-69.

[11] Y.Jiang,Y.Tang,Q. Chen, H. Liu, J. Tang, (2010) "Interval-valued intuitionistic fuzzy soft sets and their properties", Computers and Mathematics with Applications, Vol. 60, pp906-918.

[12] P.K.Maji,R.Biswas \& A.R. Roy,(2001) "Fuzzy soft sets”, J. Fuzzy Math., Vol. 9, pp589-602.

[13] P.K.Maji,R.Biswas \& A.R. Roy,(2001) "Intuitionistic fuzzy soft sets", J. Fuzzy Math., Vol. 12, pp677-692.

[14] P.K.Maji,R.Biswas \& A.R. Roy,(2003) "Soft set theory", Comp. Math. Appl., Vol. 45 pp555-562.

[15] D.Molodtsov,(1999) "Soft set theory-first results", Comp. Math. Appl., Vol. 37, pp19-31.

[16] A.Mukherjee \& A.K.Das,(2014)“Parameterized Topological Space Induced by an Intuitionistic Fuzzy Soft Multi Topological Space", Ann. Pure and Applied Math., Vol. 7, pp7-12.

[17] A.Mukherjee \& A.K.Das,(2015)“Some results on fuzzy soft multi sets”, Int. J. Cybernetics \& Informatics., Vol. 4, pp51-65.

[18] A.Mukherjee \& A.K.Das,(2013)“Topological structure formed by fuzzy soft multisets”, Rev. Bull. Cal. Math. Soc., Vol. 21, No.2, pp193-212.

[19] A.Mukherjee,A.Saha \& A.K. Das,(2013) "Interval-valued intuitionistic fuzzy soft multi set and their relations”, Ann. Fuzzy Math. Inform., Vol. 6, pp781-798.

[20] A.Mukherjee,A.K. Das \& A. Saha, (2014) "Topological structure formed by soft multi sets and soft multi compact space", Annals of Fuzzy Mathematics and Informatics, Vol. 7, pp919-933

[21] D.Tokat \& I. Osmanoglu,(2011)“Soft multi set and soft multi topology”, Nevsehir Universitesi Fen Bilimleri Enstitusu Dergisi Cilt., Vol. 2, pp109-118.

[22] L.A.Zadeh,(1965)“Fuzzy sets”,Inform. Control.,Vol. 8,pp338-353.. 